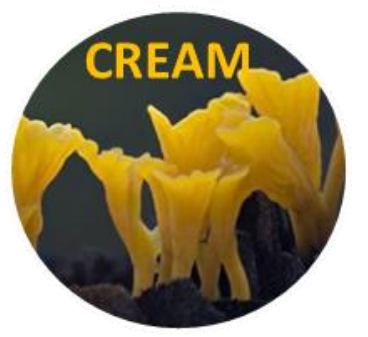

Current Research in Environmental \& Applied Mycology 5 (4): 322-348(2015) ISSN 2229-2225

$\underline{\text { www.creamjournal.org }}$

Article

CREAM

Copyright $@ 2015$

Doi 10.5943/cream/5/4/3

Online Edition

\title{
A Checklist of Coprophilous Agarics of India
}

\author{
Amandeep $\mathrm{K}^{1^{*}}$, Atri NS ${ }^{2}$ and Munruchi $\mathrm{K}^{2}$ \\ ${ }^{1}$ Bhai Gurdas Institute of Education, Sangrur-148001, Punjab, India. \\ ${ }^{2}$ Department of Botany, Punjabi University, Patiala-147002, Punjab, India.
}

Amandeep K, Atri NS, Munruchi K 2015 - A Checklist of Coprophilous Agarics of India. Current Research in Environmental \& Applied Mycology 5(4), 322-348, Doi 10.5943/cream/5/4/3

\begin{abstract}
This checklist consists of 135 species belonging in 27 genera and 10 families of the Order Agaricales, Class Agaricomycetes, and Phylum Basidiomycota. The families, genera and species have been arranged alphabetically. The status and taxonomic placement of each taxon included in the list has been updated as per the information available on the Index Fungorum/MycoBank. At the family level, Psathyrellaceae has the highest number of coprophilous species (46) reported from India, followed by Agaricaceae and Bolbitiaceae (29 spp. each) and Strophariaceae (20 spp.). The ten most represented coprophilous genera are Conocybe (23 spp.), Panaeolus (19 spp.), Coprinopsis (14 spp.), Psilocybe (13 spp.), Agaricus (9 spp.), Lepiota and Psathyrella (6 spp. each), Coprinellus and Coprinus (5 spp. each) and Bolbitius (4 spp.). The geographical distribution of the species covers 13 States (Assam, Bihar, Gujarat, Himachal Pradesh, Jammu \& Kashmir, Karnataka, Kerala, Maharashtra, Orissa, Punjab, Tamil Nadu, Uttar Pradesh and West Bengal) and 2 Union Territories (Chandigarh, New Delhi) of India. The relevant information is based on the survey of dung localities in Punjab state during the period 20072011 and original information contained in 97 research papers. The checklist is an attempt to provide updated information regarding the diversity of coprophilous agarics in India.
\end{abstract}

Key words - Agaricales - Agaricomycetes - Basidiomycota - biodiversity - dung - Punjab

\section{Introduction}

India is located in the southern part of Asia and borders the Arabian Sea, the Indian Ocean, and the Bay of Bengal. It occupies most of the South Asian continent and consists of twenty-nine states and seven union territories covering an area of 3,287,590 square kilometers. The country is situated north of the equator between $8^{\circ} 4^{\prime}$ and $37^{\circ} 6^{\prime}$ north latitude and $68^{\circ} 7^{\prime}$ and $97^{\circ} 25^{\prime}$ east longitude. It experiences a variety of climatic conditions due to its great size and varying altitude. The climate ranges from tropical in the south to temperate and alpine in the Himalayan north, where elevated regions receive sustained winter snowfall. The inland of the peninsula ranges from subtropical to temperate. The coasts of the peninsula are humid and tropical. Its four seasons are determined by monsoons, a pattern of winds sweeping across southern Asia. There is a dry, cool season (winter) from December through March; a hot season (spring) in April and May; the rainy season (summer) from June through September; and a less-rainy season (autumn) in October and November. Temperatures for the entire nation reach an average high of $38^{\circ}$ to $40^{\circ} \mathrm{C}\left(100^{\circ}\right.$ to $\left.104^{\circ} \mathrm{F}\right)$ and dip to an average low of $10^{\circ} \mathrm{C}\left(50^{\circ} \mathrm{F}\right)$. Rainfall for the entire India averages 105 centimeters (41 inches). Around $23.68 \%$ of India is covered by forests. Various domesticated and wild herbivores are found on the grazing lands of the country. Of the total 
livestock, about $90 \%$ are cattle and buffaloes and the rest sheep, goats, elephants, nilgais, camels, and rabbits (Balfour 1976, Wolpert 1999, http://www.123india.com accessed on March 31, 2015).

The very high biological diversity of India is primarily due to the highly diversified ecological niches. It is obvious from the above description that India must be harbouring diverse mycoflora. The marked variation in climate plays a determinate role in growth and development of a wide variety of mushrooms including coprophilous mycoflora. In view of this, an attempt has been made to compile the diversity of coprophilous agarics of India.

\section{Materials \& Methods}

This checklist contains information gathered partly from a study conducted on coprophilous mushrooms from the state of Punjab during the years 2007-2011 and partly from published sources up to $31^{\text {st }}$ March, 2015.

The study area in Punjab constitutes $1.57 \%$ of the total area of the country. The amount of rainfall ranges between $250 \mathrm{~mm}$ to $1000 \mathrm{~mm}$ in the state. About $70-80 \%$ of the total rainfall is concentrated during July, August and September and the rest occurs during the winter months. Punjab is primarily an agrarian state having diverse flora and fauna. It has seven to eight months of mean monthly temperature of more than $20^{\circ} \mathrm{C}$. The state has been explored systematically to ascertain the diversity, seasonal availability, distribution and ecology of coprophilous mushrooms. In this work, 172 collections belonging to 95 species spread over 20 genera of 7 agaric families are cited as a result of the study.

Besides the study, relevant information about Indian coprophilous mushrooms is searched from all forms of published documentation and literature.

The checklist is done by compiling all the available data. The names of some species as reported in the cited publications have been replaced by currently accepted name according to the MycoBank (www.mycobank.org)/Species Fungorum (www.speciesfungorum.org) websites and this has been indicated in such cases and listed in Table 1 . The checklist is organized alphabetically by family, genus and species. The distribution of species is cited according to the state where it was collected.

\section{Checklist of Taxa}

I. Family: Agaricaceae Chevall. in Flore Générale des Environs de Paris p. 121, 1826.

Type genus: Agaricus L.: Fr.

(i) Genus: Agaricus L.: Fr. in Species Plantarum 1: 1171, 1753.

Type species: Agaricus campestris L.

1. Agaricus brunnescens Peck in Bull. Torrey Bot. Club 27: 16, 1900.

West Bengal: (Pal \& Mukherjee 1977).

Himachal Pradesh: Kasauli (Sehgal 1978).

Jammu \& Kashmir: (Agha 1978, Kaul 1978).

Maharashtra: Poona (Sathe \& Deshpande 1980).

Punjab: growing scattered on manure heaps along roadsides (Srivastava 1978, Kannaiyan \& Ramasamy 1980, Saini \& Atri 1995).

2. Agaricus campestris L. in Species Plantarum 1: 1173, 1753.

Jammu \& Kashmir: Pang Range (Hennings 1900), Pehlgam (Watling \& Gregory 1980).

West Bengal: Calcutta (Bose \& Bose 1940, Banerjee 1947).

Bihar: (Vasudeva 1960).

Maharashtra: Nagpur (Trivedi 1972).

Uttar Pradesh: Allahabad (Singh \& Mehrotra 1974). 
Punjab: Sangrur $(231 \mathrm{~m})$, Qila Rehmatgarh, growing in caespitose groups on mixed cattle dung, Amandeep Kaur, PUN 4772, June 22, 2008; Ferozepur (182 m), Sodhinagar, growing scattered on buffalo dung, Amandeep Kaur, PUN 4773, August 16, 2011.

3. Agaricus cupreobrunneus (Schäffer \& Steer: Møller) Pilát in Acta Mus. Nat. Pragae 7 (1):14, 1951. Maharashtra: (Patil et al. 1995).

Punjab: Sangrur $(231 \mathrm{~m})$, Fatehgarh Channa, growing scattered in a fairy ring on mixed cattle dung alongwith earthworm excreta, Amandeep Kaur, PUN 4213, July 25, 2010 (Amandeep et al. 2015b).

4. Agaricus flavistipus Atri, M. Kaur and A. Kaur in Kavaka 42: 22, 2014.

Punjab: Faridkot (196 m), Swaag, growing solitary on buffalo dung, Amandeep Kaur, PUN 4774, August 19, 2011(Kaur et al. 2014b).

5. Agaricus halophilus Peck in Bull. New York State Mus. 94: 86, 1905.

Punjab: Mohali (316 m), Dau Majra, growing scattered on mixed cattle dung heap, Amandeep Kaur, PUN 4211, July 10, 2010; Sangrur (231 m), Daulatpur Channa, growing in groups on buffalo dung, Amandeep Kaur, PUN 4212, July 25, 2010 (Amandeep et al. 2015b).

6. Agaricus placomyces Peck in Ann. Report on the New York St. Mus. of Nat. Hist. 29: 40, 1878.

South-Western India: (Sathe \& Rahalkar 1978).

Himachal Pradesh: Solan (Sharma et al. 1978).

Punjab: (Saini et al. 1991); Patiala (251m), Chhat Bir, growing solitary on deer dung, Amandeep Kaur, PUN 4775, September 19, 2011.

7. Agaricus pratensis Schaeff. in Fung. Bavar. Palat. Nasc. 4: 42, 1774.

Assam: Guwahati (Bhattacharya \& Baruah 1953).

Punjab: Patiala $(251 \mathrm{~m})$, it growing on soil along the roadside (Saini et al. 1991); Sangrur (231m), Jamalpura, growing in groups on mixed cattle dung, Amandeep Kaur, PUN 4210, June 22, 2008.

8. Agaricus stellatus-cuticus Atri, M. Kaur and A. Kaur in Kavaka 42: 20, 2014.

Punjab: Sangrur $(231 \mathrm{~m})$, Qila Rehmatgarh, growing solitary on sheep dung among plant debris, Amandeep Kaur, PUN 4776, September 19, 2011 (Kaur et al. 2014b).

9. Agaricus xanthodermus Genevier in Bull. Soc. Bot. France 23: 31, 1876.

Punjab: Sangrur $(231 \mathrm{~m})$, Sandaur, growing scattered on mixed cattle dung, Amandeep Kaur, PUN 4777, September 28, 2008; AahanKhaeri, growing scattered on mixed cattle dung, Amandeep Kaur, PUN 4778, June 27, 2011.

(ii) Genus: Chlorophyllum Massee in Bull. Misc. Inf. Roy. Bot. Gard. Kew 189: 136, 1898.

Type species: Chlorophyllum esculentum Massee

10. Chlorophyllum humei (Murrill) Vellinga in Mycotaxon 83: 416, 2002.

Punjab: Patiala $(251 \mathrm{~m})$, Shekhpura, growing gregariously on mixed cattle dung, Babita Kumari, PUN 4112, May 23, 2008; Punjabi University, growing scattered on mixed cattle dung, Babita Kumari, PUN 4110, May 27, 2008; Behal, growing in groups on mixed cattle dung, Amandeep Kaur, PUN 4065, June 16, 2008; Sangrur (231 m), Lasoi, growing in groups on manured ground, Amandeep Kaur, PUN 4207, June 17, 2008; Barnala (228 m), Sherpur, growing solitary on buffalo dung, Amandeep Kaur, PUN 4066, June 26, 2008; Chhat Bir (251 m), growing solitary on buffalo dung, Amandeep Kaur, PUN 4067, June 30, 2008; Patiala (251 m), Punjabi University, growing scattered on cattle dung, Babita Kumari, PUN 4113, July 16, 2008; Punjabi University, growing gregariously on cattle dung, Babita Kumari, PUN 4115, August 12, 2008; Ludhiana (254 m), Seora, growing in groups on manured soil near mixed cattle dung heap, Amandeep Kaur, PUN 4068, July 25, 2009; Bathinda (251 m), Jassi Pau 
Wali, growing in groups on mixed cattle dung, Amandeep Kaur, PUN 4069, August 02, 2009; Patiala (251 m), Bahadurgarh, growing scattered on cattle dung, Babita Kumari, PUN 4097, August 18, 2009; Rajpura, growing in groups on cattle dung, Babita Kumari, PUN 4103, August 19, 2009.

North West India: Atri et al. (2014) as Lepiota humei.

11. Chlorophyllum molybdites (G. Mey.) Massee in Bull. Misc. Inf. Roy. Bot. Gard. Kew 189: 136, 1898.

Uttar Pradesh: Allahabad (Singh \& Mehrotra 1974); Lucknow (Ghosh et al. 1976).

Maharashtra: (Sathe \& Deshpande 1980).

Tamil Nadu: Madras, Deer Park (Manjula 1980, Natarajan \& Manjula 1981).

Kerala: growing scattered or in fairy rings in fields, around the basins of plants where manuring is done (Bhavani Devi 1995).

Punjab: Sangrur $(231 \mathrm{~m})$ : Qila Rehmatgarh, growing scattered in a group on camel dung, Amandeep Kaur, PUN 4779, September 27, 2010; Hoshiarpur (295 m), Jattpur, growing gregariously on buffalo dung, Amandeep Kaur, PUN 4780, July 05, 2011; Ropar (394 m), Bairampur, growing scattered on mixed cattle dung heap, Narinderjit Kaur, PUN 4682, August 22, 2011.

12. Chlorophyllum rhacodes (Vittad.) Vellinga in Mycotaxon 83: 416, 2002.

The species was earlier reported from India by Butler \& Bisby (1931) Sharma et al. (1978) and Pushpa \& Purushothama (2012) as Macrolepiota rhacodes. The mushroom has been transferred to Chlorophyllum rhacodes by Vellinga (2002). According to MycoBank/ Species Fungorum, Macrolepiota rhacodes is a synonym of Chlorophyllum rhacodes which is a valid species. Thus Macrolepiota rhacodes stands deleted from the Indian record.

Uttar Pradesh: Kalsia Hills (Butler \& Bisby 1931) as Macrolepiota rhacodes.

Himachal Pradesh: Solan (Sharma et al. 1978) as Macrolepiota rhacodes.

Punjab: Moga, Chak Fatehpur, growing in a fairy ring on buffalo dung, Amandeep Kaur, PUN 4781, June 28, 2011.

Karnataka: (Pushpa \& Purushothama 2012) as Macrolepiota rhacodes.

(iii) Genus: Coprinus Pers. in Tent. Disp. Meth. Fung. p. 62, 1797.

Type species: Coprinus comatus (Müll.: Fr.) Gray

The molecular studies by Readhead et al. (2001) have resulted in splitting erstwhile Coprinus genus belonging to family Coprinaceae into four genera, namely Coprinus sensu stricto, Coprinopsis P. Karst, Coprinellus P. Karst. and Parasola Redhead, Vilgalys \& Hopple. The coprinoid genera Coprinopsis, Coprinellus and Parasola have been shifted to the family Psathyrellaceae while genus Coprinus sensu stricto has been shifted to family Agaricaceae because of phylogenetic proximity. As per MycoBank record, the family name Coprinaceae has been placed as synonym of family Agaricaceae. Hence, Coprinaceae stands deleted from Indian record.

13. Coprinus comatus var. caprimammillatus Bogart in Mycotaxon 4(1): 274, 1976.

Punjab: Sangrur $(231 \mathrm{~m})$, Qila Rehmatgarh, solitary to densely grouped on mixed cattle dung in a pasture, Amandeep Kaur, PUN 4061, June 03, 2008; Bathinda (211 m), Naruaana, growing in groups on mixed cattle dung, Amandeep Kaur, PUN 4782, August 01, 2009 (Amandeep et al. 2015b).

14. Coprinus comatus var. comatus (Müll.: Fr.) Gray in Nat. Arrang. Brit. Pl. 1: 632, 1821.

West Bengal: Darjeeling (Berkeley 1851); Calcutta (Banerjee 1947).

Gujarat: Baroda (Moses 1948).

Maharashtra: Bombay (Berkeley 1851); Nagpur (Trivedi 1972).

Uttar Pradesh: Lucknow (Ghosh et al. 1974); Allahabad (Singh \& Mehrotra 1974). 
Jammu \& Kashmir: Dachigan Reserve (Watling \& Gregory 1980); Kashmir Valley (Kaul \& Kachroo 1974).

Punjab: Patiala (251m), Chhatbir, growing on horse dung (Kaushal \& Grewal 1992, Saini \& Atri 1995); Moga (217 m), Ajitwal, growing scattered on mixed cattle dung, Amandeep Kaur, PUN 4783, August 13, 2010.

15. Coprinus filiformis Berk. \& Br. in Ann. Mag. Nat. Hist. 7: 379, 1861.

Punjab: growing on dung of nilgai (Rea 1922, Ginai 1936, Saini \& Atri 1995).

16. Coprinus papillatus (Batsch) Fr. in Elenchus Fungorum Continuatio Prima p. 81, 1838.

Punjab: growing on sambhar dung (Rea 1922, Mahju 1933, Saini \& Atri 1995); Chhatbir, growing on panther dung (Kaushal \& Grewal 1992, Saini \& Atri 1995).

17. Coprinus sterquilinus (Fr.) Fr. in Epicrisis Systematis Mycologici p. 242, 1838.

Jammu \& Kashmir: Srinagar, Sanat Nagar (Watling \& Gregory 1980).

Punjab: Ludhiana (254 m), Lohatbaddi, growing solitary on horse dung, Amandeep Kaur, PUN 4785, June 10, 2008; Faridkot (196 m), Bajakhana, growing solitary on manured soil, Harwinder Kaur, PUN 4771, September 10, 2009; Sangrur (231 m), Sikanderpura, growing scattered on mixed cattle dung, Amandeep Kaur, PUN 4786, June 29, 2011.

(iv) Genus: Crucispora E. Horak in New Zealand Journal of Botany 9: 489, 1971.

Type species: Crucispora naucorioides E.Horak

18. Crucispora rhombisperma (Hongo) E. Horak in Sydowia 33: 57, 1980.

Kerala: Wayanad, Ponkuzhy, growing scattered on elephant dung, K. A. Thomas T338, October 17, 1999, K. A. Thomas T338b, October 18, 1999, K. A. Thomas T338c, October 31, 1999, K. A. Thomas T338d (Manimohan et al. 2007, Noordeloos et al. 2007) as Panaeolina rhombisperma.

(v) Genus: Lepiota (Pers. ex Fr.) S.F. Gray in Nat. Arrang. Brit. Plants, 1: 601, 1821.

Type species: Lepiota colubrina (Pers. ex Fr.) S.F. Gray

19. Lepiota epicharis var. occidentalis Dennis in Kew Bull. 15: 111, 1962.

Tamil Nadu: Madras, Guindy, Raj Bhavan (Manjula 1980, 1983).

Punjab: Hoshiarpur (295 m), Shehbazpur Tanda, growing in a group on mixed buffalo dung and wheat straw heap, Munruchi Kaur and Amandeep Kaur, PUN 4787, September 03, 2011 (Amandeep et al. 2015b).

20. Lepiota leprica (Berk. \& Br.) Sacc. in Syll. Fung. 4: 56, 1887.

North-East Hills: growing solitary or in groups in open fields and pastures on cow dung or organic matter rich soil (Verma et al. 1995).

21. Lepiota subincarnata J.E. Lange in Flora Agaricina Danica 5: 5, 1940.

Kerala: (Kumar \& Manimohan 2009).

Punjab: Sangrur (231 m), Qila Rehmatgarh, growing solitary on camel dung, Amandeep Kaur, PUN 4788, September 19, 2011(Amandeep et al. 2015b).

22. Lepiota thiersii Sundberg in Mycotaxon 34 (1): 242, 1989.

Punjab: (Atri et al. 2000); Mohali (316 m), Bhajauli, growing in groups on buffalo dung heap, Amandeep Kaur, PUN 4070, August 21, 2009.

23. Lepiota thrombophora (Berk. \& Br.) Sacc. in Syll. Fung. 5: 53, 1887.

Tamil Nadu: Madras, IIT campus (Manjula 1980, 1983). 
Punjab: Ropar (394 m): Mugal Majri, growing in groups on mixed cattle dung, Amandeep Kaur, PUN 4071, August 21, 2009 (Amandeep et al. 2015b).

24. Lepiota xanthophylla P.D. Orton in Trans. Brit. Mycol. Soc. 43(2): 289, 1960.

Punjab: Faridkot (196 m), Deena Kangar, growing scattered on buffalo dung, Amandeep Kaur, PUN 4789, August 19, 2011(Amandeep et al. 2015b).

(vi) Genus: Leucoagaricus (Locquin) Sing. in Sydowia 2: 35, 1968.

Type species: Leucoagaricus macrorhizus (Locquin) Sing.

25. Leucoagaricus leucothites (Vittad.) Wasser, Ukr. bot. Zh. 34(3): 308, 1977.

Trivedi (1972), Singh \& Mehrotra (1974), Manjula (1980, 1983), Bhavani Devi (1995) recorded the species from India as Leucoagaricus naucinus. According to Index Fungorum and Kew Mycology (2013), the current name of Leucoagaricus naucinus is Leucoagaricus leucothites.

Maharashtra: Nagpur (Trivedi 1972) as Leucoagaricus naucinus.

Uttar Pradesh: Allahabad (Singh \& Mehrotra 1974) as Leucoagaricus naucinus.

Tamil Nadu: Madras, Guindy, Deer Park, growing on elephant dung (Manjula 1980, 1983) as Leucoagaricus naucinus.

Kerala: growing scattered on the ground in pastures and fields (Bhavani Devi 1995) as Leucoagaricus naucinus.

Punjab: Hoshiarpur (295 m), Jejon Duaba, growing gregariously forming a fairy ring on mixed cattle dung heap, Amandeep Kaur, PUN 4790, July 05, 2011.

26. Leucoagaricus meleagris (Gray) Singer in Lilloa 22: 422, 1951.

Uttar Pradesh: Saharanpur (Hennings 1901) as Lepiota meleagris.

Orissa: growing solitary or in caespitose clusters on cow dung (Dhancholia \& Sinha 1990) as Lepiota meleagris.

(vii) Genus: Leucocoprinus Pat. in Journal de Botanique (Morot) 2:16,1888.

Type species: Leucocoprinus cepistipes (Sowerby) Patouillard

27. Leucocoprinus cepistipes (Sowerby) Pat. in Journal de Botanique (Morot) 3: 336, 1889.

Maharashtra: Poona (Massee 1901) as Lepiota cepistipes; (Sathe \& Deshpande 1980).

West Bengal: Calcutta (Bose 1920, Banerjee 1947) as Lepiota cepistipes.

Maharashtra: Bombay, on dung (Patel \& Kamat 1935) as Lepiota sordescens.

Tamil Nadu: Madras (Manjula 1980).

Orissa: growing on cow dung (Dhancholia \& Sinha 1990).

Kerala: growing in caespitose on the manured ground on humus rich soil (Bhavani Devi 1995).

Punjab: Ropar $(394 \mathrm{~m})$, Bhupnagar, growing scattered on mixed cattle dung heap, Amandeep Kaur, PUN 4208, July 10, 2010; Jallandhar (233 m), Kala Bakkra, growing in groups on buffalo dung, Amandeep Kaur, PUN 4209, July 30, 2010; Hoshiarpur (295 m): Mehlanwaali, growing in groups on mixed cattle dung, Amandeep Kaur, PUN 4791, July 05, 2011; Hoshiarpur (295 m), Jattpur, growing scattered on mixed cattle dung under Acacia nilotica, Narinderjit Kaur, PUN 4690, August 16, 2011; Hoshiarpur (295 m), Chaggran, growing scattered on mixed cattle dung, Narinderjit Kaur, PUN 4691, August 16, 2011.

Karnataka: Bangalore (Pushpa \& Purushothama 2011).

28. Leucocoprinus cretaceus (Bull.) Locq. in Bull. mens. Soc. linn. Soc. Bot. Lyon 14: 93, 1945.

Punjab: Chandigarh $(321 \mathrm{~m})$, growing on manure heaps and heavily manured beds (Rawla et al. 1982, Saini \& Atri 1995) as Leucocoprinus cretatus. 
29. Leucocoprinus straminellus (Bagl.) Narducci \& Caroti in Mem. Soc. Tosc. Sci. Nat. 102: 49, 1995. Punjab: Patiala (251 m), Harpalpur, growing in groups on buffalo dung heap, Amandeep Kaur, PUN 4792, July 19, 2011(Amandeep et al. 2015b).

II. Family: Amanitaceae R. Heim: Pouzar in Ceská Mykol. 37(3): 173, 1983.

Type genus: Amanita Pers.

(viii) Genus: Amanita Pers. in Neues Mag. Bot. 1: 145, 1794.

Type species: Amanita muscaria (L.) Lam.

30. Amanita solitaria (Bull.) Mérat in Nouvelle Flore des Environs de Paris 1: 121, 1836.

Kerala: growing solitary or in groups on compost or on cow dung heaps (Bhavani Devi 1995).

III. Family: Bolbitiaceae Sing. in Pap. Michigan Acad. Sci. 32:147, 1948.

Type genus: Bolbitius Fr.

(ix) Genus: Bolbitius Fr. in Epicrisis p. 253, 1838.

Type species: Bolbitius vitellinus (Pers.) Fr.

31. Bolbitius coprophilous (Peck) Hongo in Mem. Fac. Lib. Arts Educ., Shiga University, Nat. Sci. 9: 82, 1959.

Kerala: Thrissur, Guruvayur, growing on elephant dung, A. Thomas T312, June 11, 1999, A. Thomas T312b, June 17, 1999; Kasaragod, Adhoor, A. Thomas T312c, September 16, 2000 (Thomas et al. 2001, Manimohan et al. 2007).

Punjab: growing in open areas among grasses (Atri et al. 1992); Sangrur (231 m), Bamaal, growing gregariously on buffalo dung flakes near village pond, Amandeep Kaur, PUN 4793, July 22, 2009; Haidernagar, growing scattered on buffalo dung, Amandeep Kaur, PUN 4794, June 29, 2011 (Amandeep et al. 2013b).

32. Bolbitius demangei (Quél.) Sacc. \& Sacc. in Sylloge Fungorum 17: 74, 1905.

Punjab: Sangrur $(231 \mathrm{~m})$ : Binzoki, growing in groups on buffalo dung and rotting Jute fabric, Amandeep Kaur, PUN 4795, June 29, 2011; Faridkot (196 m): Chandwaja, growing in a group on mixture of cattle dung and straw, Amandeep Kaur, PUN 4796, August 19, 2011(Amandeep et al. 2013b).

33. Bolbitius marginatipes Zeller in Mycologia 25 (5): 378, 1933.

Punjab: Sangrur $(231 \mathrm{~m})$ : Haidernagar, growing scattered on buffalo dung, Amandeep Kaur, PUN 4798, June 29, 2011(Amandeep et al. 2013b).

34. Bolbitius titubans (Bull.) Fr. in Epicrisis Systematis Mycologici p. 254, 1838.

Kerala: growing scattered in coprophilous habitats Bhavani Devi (1995) as Bolbitius vitellinus.

Punjab: growing on horse dung (Rea 1922, Mahju 1933, Saini \& Atri 1995) as Bolbitius vitellinus; Sangrur (251 m), Ratolan, growing solitary on buffalo dung, Amandeep Kaur, PUN 3896, September 29, 2008 (Atri et al. 2009); Sangrur (231 m), Alipur, growing solitary on buffalo dung heap, Amandeep Kaur, PUN 4214, June 08, 2008 (Amandeep et al. 2013b) as Bolbitius vitellinus.

(x) Genus Conocybe Fayod in Ann. Sci. Nat. Bot. 9: 357, 1889.

Type species: Conocybe tenera (Schaeff.) Fayod

35. Conocybe ambigua Watling, in Notes R. bot. Gdn Edinb. 38(2): 331, 1980.

Tamil Nadu: Madras, Maduravoyal, Madras University Botany Field Research Laboratory, growing in groups on cow dung, Herb. Mubl No. 2594, November 07, 1978 (Natarajan \& Raman 1983, 1984). 
36. Conocybe antipus (Lasch) Fayod in Annls Sci. Nat., Bot., sér. 79: 357, 1889.

Kerala: growing solitary or in caespitose clusters on manure and compost heaps (Bhavani Devi 1995) as Galera antipus.

37. Conocybe apala (Fr.) Arnolds in Persoonia 18(2): 225, 2003.

Punjab: Tarn Taran, Baath (169 m), growing solitary on buffalo dung, Amandeep Kaur, PUN 4219, July 31, 2010; Tarn Taran, Naushehra Pannua (169 m), growing in groups on buffalo dung, Amandeep Kaur, PUN 4220, August 02, 2010; Ferozepur, Makhu (182 m), growing in groups on buffalo dung, Amandeep Kaur, PUN 4230, August 02, 2010; Moga (217 m), Chak Kania Wala, growing scattered on mixed cattle dung, Amandeep Kaur, PUN 4344, June 28, 2011; Hoshiarpur (295 m), Shehbaazpur Tanda, growing scattered on mixed buffalo dung and wheat straw, Amandeep Kaur, PUN 4343, September 03, 2011(Atri et al. 2012); Chhat Bir $(251 \mathrm{~m})$, growing scattered in a group on elephant dung mixed with rotten wheat straw and leaf litter, Amandeep Kaur, PUN 4799, September 19, 2011(Amandeep et al. 2015a) as Conocybe albipes.

38. Conocybe brachypodii (Velen.) Hauskn. \& Svrček in Czech Mycol. 51: 43, 1999.

Punjab: Patiala (251m), Mehmoodpur Jattan, growing in groups on mixed cattle dung heap, Amandeep Kaur, PUN 3899, June 14, 2008 (Atri et al. 2009).

39. Conocybe brunneoaurantiaca K. A. Thomas, Hauskn. and Manim. in Öst. Z. Pilzk. 10: 90, 2001.

Kerala: Malappuram, Nilambur, growing on elephant dung, Nisha NVS122, August 24, 2002 (Manimohan et al. 2007).

40. Conocybe crispa (Longyear) Singer in Lilloa 22: 485, 1951.

Punjab: Sangrur $(231 \mathrm{~m})$, Upoki, growing in caespitose cluster on partially decomposed buffalo dung flake, Amandeep Kaur, PUN 3897, August 20, 2008 (Atri et al. 2009).

41. Conocybe fuscimarginata (Murrill) Singer in Beih. Nova Hedwigia 29: 210, 1969.

Punjab: Patiala (251 m): Balbehra, growing in groups on cow dung, Amandeep Kaur, PUN 4350, June 25, 2008; Barnala (228 m): Farwahi, growing in groups on mixed cattle dung, Amandeep Kaur, PUN 4349, June 26, 2008(Amandeep et al. 2015a).

42. Conocybe juniana (Velen.) Hauskn. \& Svrček, in Öst. Z. Pilzk. 8: 46, 1999. Jammu \& Kashmir: Tangmarg (Watling \& Gregory 1980) as Conocybe magnicapitata. Punjab: Ferozepur (182 m), Sodhinagar, growing solitary on buffalo dung, Amandeep Kaur, PUN 4801, August 16, 2011(Amandeep et al. 2015a) as Conocybe magnicapitata.

43. Conocybe lenticulospora Watling in Notes Roy. Bot. Gard. Edinburgh 38(2): 351, 1980.

Punjab: Ludhiana $(254 \mathrm{~m})$, Lohatbaddi, growing in groups on mixed cattle dung, Amandeep Kaur, PUN 4347, July 14, 2008(Amandeep et al. 2015a).

44. Conocybe leucopus (Kühner) Kühner \& Watling in Notes from the Royal Botanic Garden Edinburgh 40 (3): 539, 1983.

Punjab: Ferozepur (182 m), Mudki, growing scattered in a group on cow dung on ants' hill, Amandeep Kaur, PUN 4800, August 16, 2011(Amandeep et al. 2015a).

45. Conocybe microrrhiza var. coprophila Amandeep Kaur, Atri and Munruchi Kaur in Mycosphere 6(1): 29, 2015.

Punjab; Faridkot (196 m): Chandwaja, growing solitary on cow dung, Amandeep Kaur, PUN 4802, August 19, 2011(Amandeep et al. 2015a).

46. Conocybe moseri Watling in Notes Roy. Bot. Gard., Edinburgh 38(2): 342, 1980. 
Punjab: Moga (217 m), Ajitwal, growing scattered on mixed cattle dung, Amandeep Kaur, PUN 4352, August 13, 2010; Ludhiana (254 m): Lohatbaddi, growing in groups on mixed cattle dung, Amandeep Kaur, PUN 3898, July 14, 2008 (Amandeep et al. 2015a).

47. Conocybe pseudopubescens K. A. Thomas, Hauskn. \& Manimohan in Öst. Z. Pilzk. 10: 92, 2001. Kerala: Wayanad, Muthanga, growing on elephant dung, A. Thomas T84, June 25, 1997; A. Thomas T84b, May 25, 1999 (Thomas et al. 2001, Manimohan et al. 2007).

48. Conocybe pubescens (Gillet) Kühner in Encyclop. Mycol. 7: 85, 1935.

Maharashtra: (Sathe \& Deshpande 1980).

Kerala: (Mohanan 2011).

Karnataka: Makutta Reserve forest, Virajpet, Kodagu, growing on elephant dung, N.C. Karun, MUBSNCKKRSMF \# 040, July 28, 2012 (Karun \& Sridhar 2015).

49. Conocybe rickenii (Schaef.) Kühner in Encycl. Mycol. 7: 115, 1935.

Chandigarh (231 m), growing on horse dung (Sarwal \& Rawla 1983, Saini \& Atri 1995).

Punjab: Sangrur (231 m), Jaatimajra, growing solitary on buffalo dung, Amandeep Kaur, PUN 4351, September 13, 2009 (Amandeep et al. 2015a).

50. Conocybe semiglobata Kühner \& Watling in Notes R. bot. Gdn Edinb. 38(2): 337, 1980.

Tamil Nadu: Nilgiris, Ootacamud, growing solitary on cow dung, Herb. Mubl No. 2591, November 06, 1979 (Natarajan \& Raaman 1983, 1984).

51. Conocybe subpubescens P. D. Orton in Trans. of British Mycol. Soc., 43: 195, 1960.

Punjab; Hoshiarpur $(295 \mathrm{~m})$ : Jalota Dasuya, growing scattered in a group on mixed horse dung and cattle dung heap mixed with leaf litter, Amandeep Kaur, PUN 4348, July 14, 2010 (Amandeep et al. 2015a).

52. Conocybe subxerophytica var. brunnea Hauskn. in Österr. Z. Pilzk. 11: 74, 2002.

Punjab: Barnala (228 m), Sherpur, growing in groups on horse dung, Amandeep Kaur, PUN 4217, July 13, 2008 (Atri et al. 2012).

53. Conocybe subxerophytica var. subxerophytica Singer \& Hauskn. in Pl. Syst. Evol. 213: 98, 1992. Punjab: Patiala (251m), Samana, growing scattered on buffalo dung, Amandeep Kaur, PUN 4216, June 25, 2008 (Atri et al. 2012).

54. Conocybe uralensis Hauskn., Knudsen \& Mukhin in Folia Cryptog. Estonica, Fasc. 45: 33, 2009. Punjab; Ropar (394 m): Kuraali, growing in groups on buffalo dung heap, Amandeep Kaur, PUN 4218, August 21, 2009 (Atri et al. 2012).

55. Conocybe velutipes (Velen.) Hauskn. \& Svrček in Czech Mycology 51(1): 68, 1999.

Punjab; Moga (217 m): Ajitwal, growing scattered on cow dung, Amandeep Kaur, PUN 4803, August 13, 2010 (Amandeep et al. 2015a).

56. Conocybe volvata K. A. Thomas, Hauskn. and Manim. in Öster. Z. Pilzk. 10: 101, 2001.

Kerala: Thrissur, Guruvayur, growing on elephant dung, A. Thomas T302b, June 11, 1999, A. Thomas T302c, June 17, 1999, A. Thomas T302d, July 14, 1999, A. Thomas T302e, August 21, 2000 (Thomas et al. 2001, Manimohan et al. 2007).

57. Conocybe zeylanica (Petch) Boedijn in Sydowia 5(3-6): 223, 1951.

Kerala: growing on a heap of dried cow dung (Thomas et al. 2001). 
(xi) Genus: Pholiotina Fayod in Annales des Sciences Naturelles Botanique 9: 359,1889.

Type species: Pholiotina blattaria (Fr.) Fayod

58. Pholiotina indica K. A. Thomas, Hauskn. and Manim. in Öst. Z. Pilzk. 10: 109, 2001.

Kerala: Wayanad, Muthanga, growing on elephant dung, A. Thomas T96, July 04, 1997, A. Thomas T96c, August 18, 1997, A. Thomas T179, October 01, 1997, A. Thomas T179b, June 30, 1998, A. Thomas T179f, June 09, 1999, Ponkuzhy, A. Thomas T179c, October 13, 1998, A. Thomas T179d, October 27, 1998, A. Thomas T179e, November 19, 1998; Idukki, Munar, A. Thomas T179h, October 11, 1999, A. Thomas T179i, October 12, 1999 (Thomas et al. 2001, Manimohan et al. 2007); (Mohanan 2011).

59. Pholiotina plumbeitincta (G.F. Atk.) Hauskn., Krisai \& Voglmayr in Öst. Z. Pilzk. 13: 212, 2004. Tamil Nadu: Madras, Maduravoyal, Madras University Botany Field Research Laboratory, growing in groups on cow dung, Herb. Mubl No. 2598, November 07, 1978 (Natarajan \& Raaman 1983, 1984) as Conocybe plumbeitincta.

IV. Family: Entolomataceae Kotlába \& Pouzar in Ceská Mykol. 26(4): 218, 1972.

Type genus: EntolomaFr.: Kummer

(xii) Genus: Entoloma Fr.: Kummer in Der Führer in die Pilzkunde 23, 97, 1871.

Type species: Entoloma sinuatum (Bull. ex Pers.) P. Kumm.

60. Entoloma anamikum Manim., A. V. Joseph \& Leelav. in Mycol. Res. 99 (9): 1091, 1995.

Kerala: Thrissur, Guruvayur, growing on elephant dung, Nisha NVS 136, October 28, 2002, Nisha NVS 136c, July 02, 2003; Malappuram, Nilambur, Nisha NVS 136b, June 28, 2003 (Thomas et al. 2001, Manimohan et al. 2007).

(xiii) Genus: Rhodocybe Maire in Bulletin de la Société Mycologique de France 40 (3): 298, 1926.

Type species: Rhodocybe caelata (Fr.) Maire

61. Rhodocybe popinalis var. macrosporus Amandeep Kaur, NS Atri \& Munruchi Kaur in JNBR 2(3): 261, 2013.

Punjab: Hoshiarpur (295 m), Asalpur, growing in caespitose groups on mixed cattle and horse dung heap, Amandeep Kaur, PUN 4804, July 14, 2010 (Kaur et al. 2013a).

V. Family: Lyophyllaceae Jülich in Biblioth. Mycol. 85: 378, 1981.

Type genus: Lyophyllum P. Karst.

(xiv) Genus: Termitomyces R. Heim in Arch. Mus. Nat. Hist. Ser. 6 18:147, 1942.

Type species: Termitomyces striatus (Beeli) Heim

62. Termitomyces radicatus Natarajan in Curr. Sci. 46: 679, 1977.

Jammu \& Kashmir: Jammu (Natarajan 1977).

Kerala: (Pegler \& Vanhaecke 1994).

Punjab: Patiala $(251 \mathrm{~m})$, growing on sandy or humicolous soil (Atri et al. 2005); Sangrur (231 m), Dohla, growing gregariously on cattle manured soil along road side, Amandeep Kaur, PUN 4805, August 19, 2010; Ferozepur (182 m), Badaduraka, growing gregariously in caespitose groups on mixed cattle dung among grasses, Amandeep Kaur, PUN 4806, August 16, 2011.

VI. Family: Mycenaceae Overeem in Icon. Fung. Malay.: 14, 1926.

Type genus: Mycena (Pers.) Roussel 
(xv) Genus: Mycena (Pers.) Roussel in Fl. Calvados, Edn 2: 64 ('46'), 1806.

Type species: Mycena galericulata (Scop.) Gray

63. Mycena glatfelteri (Peck) Murrill in N. Amer. Fl. (New York) 10(3): 190, 1917.

Punjab: Patiala $(251 \mathrm{~m})$, Bhedpura, growing scattered in a group on buffalo dung and leaf litter, Amandeep Kaur, PUN 4797, July 16, 2011 (Amandeep et al. 2013b as Bolbitius glatfelteri).

VII. Family: Pluteaceae Kotl. \& Pouzar in Ceská Mykologie 26(4): 218, 1972.

Type genus: Pluteus Fr.

(xvi) Genus: Volvariella Speg. in Fungi Argentini Novi Vel Critici p. 119, 1898.

Type species: Volvariella argentina Speg.

64. Volvariella hypopithys (Fr.) Shaffer in Mycologia 49(4): 572, 1957.

Kerala: (Pradeep et al. 1998)

Punjab: (Saini et al. 1983); Sangrur (231 m), Langrian, growing scattered on buffalo dung, Amandeep Kaur, PUN 4215, June 21, 2008.

65. Volvariella pusilla (Pers.) Singer in Lilloa 22: 401, 1951.

Tamil Nadu: Madras (Natarajan 1978).

Uttar Pradesh: Lucknow (Ghosh et al. 1967).

Kerala: (Pradeep et al. 1998).

Punjab: (Saini et al. 2008-2009); Sangrur (231 m), Jamalpura, growing in caespitose cluters on mixed cattle and camel dung heap, Amandeep Kaur, PUN 4807, June 22, 2008.

West Bengal: (Dutta et al. 2011).

66. Volvariella volvacea (Bull.) Singer in Lilloa 22: 401, 1951.

Kerala: Malappuram, Ramapuram, growing on elephant dung, Nisha NVS 137, November 11, 2002, Nisha NVS 137b, November 13, 2002; Thrissur, Guruvayur, growing on elephant dung, Nisha NVS 137c, April 11, 2003, Nisha NVS 137d, July 02, 2003 (Manimohan et al. 2007).

(xvii) Genus: Volvopluteus Vizzini, Contu \& Justo in Fungal Biol. 115(1): 15, 2011.

Type species: Volvopluteus gloiocephalus (DC.: Fr.) Vizzini, Contu \& Justo

The genus Volvopluteus was established by Justo et al. (2010a) on the basis of molecular systematic studies. Myco Bank documents 04 species namely, V. asiaticus, V. earlei, V. gloiocephalus and $V$. michiganensis, belonging to this genus. From coprophilous habitats of India, 02 species namely Volvopluteus earlei and Volvopluteus gloiocephalus have been recorded.

67. Volvopluteus earlei (Murrill) Vizzini, Contu and Justo in Fungal Biol. 115(1):15, 2011.

This species was earlier described under the genus Volvariella as V. earlei from India by Atri et al. (1996). Based on the molecular systematic studies by Justo et al. (2010a,b), Volvariella earlei stands transferred to the genus Volvopluteus and now is a synonym of Volvopluteus earlei. In view of the above, Volvariella earlei stands replaced by Volvopluteus earlei in the record.

Punjab: Patiala, growing scattered among grasses (Atri et al. 1996) as Volvariella earlei; Patiala (251 $\mathrm{m})$, Lahore Majra, growing scattered in groups on buffalo dung, Amandeep Kaur, PUN 4808, June 23, 2008; Patiala $(251 \mathrm{~m})$ : Bhavanigarh Road, growing scattered in groups on buffalo dung, Amandeep Kaur, PUN 4809, June 23, 2008.

68. Volvopluteus gloiocephalus (DC.: Fr.) Vizzini, Contu \& Justo in Fungal Biol. 115(1): 15, 2011.

This species was earlier described under the genus Volvariella as V. speciosa from India by Ghosh et al. (1967), Pathak et al. (1978), Saini \& Atri (1993), and Bhavani Devi (1995). On the basis of 
DNA evidences, Justo et al. (2010a,b) reclassified V. speciosa as Volvopluteus gloiocephalus. The latest MycoBank record refers Volvariella speciosa and Volvariella gloiocephala as synonyms of Volvopluteus gloiocephalus. Thus Volvariella speciosa stands replaced by Volvopluteus gloiocephalus in the record.

Uttar Pradesh: Lucknow (Ghosh et al. 1967, Pathak et al. 1978) as Volvariella speciosa.

Kerala: growing solitary or gregariously on rich manured ground (Bhavani Devi 1995); (Pradeep et al. 1998) as Volvariella speciosa.

Punjab: Patiala, growing on sandy soil (Saini \& Atri, 1993 as Volvariella speciosa); Sangrur (231 m), Bhavanigarh, growing in groups on mixed cattle dung, Amandeep Kaur, PUN 4810, June 23, 2008; Hoshiarpur (295 m), Siprian, growing scattered on mixed horse and cattle dung, Amandeep Kaur, PUN 4811, July 14, 2010; Sangrur (231 m), Madevi, growing in a caespitose group on buffalo dung, Amandeep Kaur, PUN 4812, June 27, 2011.

West Bengal: growing solitary on cow dung mixed with decaying paddy straw or on compost heaps (Dutta et al. 2011) as Volvariella gloiocephala.

VIII. Family: Psathyrellaceae Vilgalys, Moncalvo \& Redhead in Taxon 50(1): 226, 2001.

Type genus: Psathyrella Fr.: Quél.

Readhead et al. (2001) performed a phylogenetic analysis of the genus Coprinus traditionally described in the family Coprinaceae and reclassified the coprinoid taxa traditionally grouped under Coprinus into four genera, namely Coprinus Pers. sensu stricto, Coprinopsis P. Karst, Coprinellus P. Karst and Parasola Redhead, Vilgalys \& Hopple into two different families. Now the genus Coprinus sensu stricto belongs to family Agaricaceae and the genera Coprinopsis, Coprinellus and Parasola to the family Psathyrellaceae Vilgalys, Moncalvo \& Redhead.

From Indian coprophilous habitats, mushrooms belonging to the different coprinoid genera namely Coprinellus, Coprinopsis and Parasola of family Psathyrellaceae were recorded from time to time and described under the genus Coprinus. This list provides updated information regarding their valid names as per MycoBank records.

(xviii) Genus: Coprinellus P. Karst. in Bidrag Kännedom of Finlands Natur Folk 32: 542, XXVIII, 1879.

Type species: Coprinellus deliquescens (Bull.) P. Karst.

As a result of the molecular studies by Hopple \& Vilgalys (1999) and Readhead et al. (2001), the coprinoid members with cellular pileus cuticle and comparatively more persistent and less wooly pileal veil remnants, have been transferred to the genus Coprinellus. Species recorded from coprophilous habitats of India and earlier described under the genus Coprinus exist as legitimate species under Coprinellus as per the latest information on MycoBank.

69. Coprinellus ephemerus (Bull.) Redhead, Vilgalys \& Moncalvo in Taxon 50(1): 233, 2001.

Punjab: growing on rabbit dung (Rea 1922, Mahju 1933, Saini \& Atri 1995) as Coprinus ephemerus; Hoshiarpur (295 m), Mehlanwaali, growing in groups on mixed cattle dung, Amandeep Kaur, PUN 4813, July 5, 2011.

70. Coprinellus fimbriatus (Berk. \& Br.) Redhead, Vilgalys \& Moncalvo in Taxon 50(1): 233, 2001. West Bengal: Howrah and Hooghly districts, on dung (Bose 1920, Manjula 1983) as Coprinus fimbriatus.

71. Coprinellus micaceus (Bull.: Fr.) Vilgalys, Hopple \& Jacq. Johnson in Taxon 50: 234, 2001.

West Bengal: Calcutta (Banerjee 1947) as Coprinus micaceus.

Maharastra: Nagpur (Trivedi 1972, Patil et al. 1995) as Coprinus micaceus.

Kerala: (Bhavani Devi 1995) as Coprinus micaceus.

Himachal Pradesh: Solan (Thapa et al. 1977, Lakhanpal 1993) as Coprinus micaceus. 
Jammu and Kashmir: Sonamarg (Kaul \& Kachroo 1974, Watling \& Gregory 1980, Abraham 1991) as Coprinus micaceus.

Assam: (Gogoi et al. 2000)

Punjab: Patiala (251 m), Bhunerheri, growing in groups on mixed dung, Amandeep Kaur, PUN 4814, June 16, 2008; Hoshiarpur (295 m), Tanda, growing in groups on humicolous, manured soil, Narinderjit Kaur, PUN 4684, July 23, 2011.

72. Coprinellus micaceus var. macrosporus Atri \& Kaur in Plant Diversity in India p. 436, 2004.

Atri \& Kaur (2004) described Coprinus micaceus var. macrosporus from India. The species Coprinus micaceus has been transferred to Coprinellus micaceus by Readhead et al. (2001) in view of which Coprinus micaceus var. macrosporus described earlier now becomes Coprinellus micaceus var. macrosporus.

Punjab: Sirhind $(250 \mathrm{~m})$, growing in clusters on cattle dung manured soil under Psidium guazava tree, Amanjeet Kaur, PUN 2960, January 12, 1999 (Atri \& Kaur 2004 as Coprinus micaceus var. macrosporus).

73. Coprinellus truncorum (Scopoli) Redhead, Vilgalys \& Moncalvo in Taxon 50(1): 235, 2001. Jammu \& Kashmir: Srinagar, Sanat Nagar (Watling \& Gregory 1980, Abraham 1991 as Coprinus truncorum).

Punjab: Ludhiana $(254 \mathrm{~m})$, Kamaalpura, growing in groups on mixed cattle dung, Amandeep Kaur, PUN 4815, January 30, 2010.

(xix) Genus: Coprinopsis P. Karst. in Acta Societatis pro fauna et flora Fennica 2(1): 27, 1881. Type species: Coprinopsis friesii (Quél.) P. Karst.

As a result of the molecular studies by Hopple \& Vilgalys (1999) and Readhead et al. (2001), the coprinoid members which possess hyphal pileus cuticle and abundant powdery to floccose veil covering the whole pileus, have been transferred to the genus Coprinopsis. As per MycoBank record following species now exist as legitimate species of genus Coprinopsis instead of Coprinus.

74. Coprinopsis cinerea (Schaeff.) Redhead, Vilgalys \& Moncalvo in Taxon 50(1): 227, 2001. Punjab: (Ginai 1936, Manjula 1983) as Coprinus cinereus; Sangrur (231 m): Sikanderpura, growing in groups on mixed cattle dung, Amandeep Kaur, PUN 4816, June 02, 2008; Patiala (251 m): Kalyan, growing in groups on horse dung, Amandeep Kaur, PUN 4817, January 31, 2010 (Amandeep et al. 2014).

75. Coprinopsis cordispora (T.Gibbs) Watling and M.J. Richardson in Edinburgh J. Bot. 67(3): 406, 2010.

Punjab: Hoshiarpur (295m): Satiana, growing in groups on mixed cattle and horse dung heap, Amandeep Kaur, PUN 4784, July 14, 2010 (Amandeep et al. 2015b).

76. Coprinopsis cothurnata var. equsterca Atri, A. Kaur \& M. Kaur in Mycosphere 5(1): 18, 2014. Punjab: Barnala (228 m), Salempur, growing in a caespitose group on horse dung, Amandeep Kaur, PUN 4064, February 08, 2009 (Amandeep et al. 2014).

77. Coprinopsis foetidella (P. D. Orton) Atri, A. Kaur \& M. Kaur in Mycosphere 5(1): 17, 2014. Punjab: Moga (217 m), Jallalabad, growing in group on buffalo dung, Amandeep Kaur, PUN 4818, June 28, 2011(Amandeep et al. 2014).

78. Coprinopsis lagopides var. lagopides (P. Karst.) Redhead, Vilgalys \& Moncalvo in Taxon 50(1): 229, 2001.

Jammu \& Kashmir: Pehlgam (Watling \& Gregory 1980, Abraham 1991) as Coprinus lagopides. 
Punjab: Sangrur (231 m), Naushehra, scattered on mixed cattle dung and straw heap, Amandeep Kaur, PUN 4060, July 9, 2007 (Amandeep et al. 2014).

79. Coprinopsis lagopus (Fr.) Redhead, Vilgalys \& Moncalvo in Taxon 50(1): 229, 2001.

New Delhi: (Krishnamurthy \& Verma 1974, Manjula 1983) as Coprinus lagopus.

Tamil Nadu: Madras, Maduravoyal, Madras University Campus, growing in groups on paddy straw, Herb. Mubl No. 2556, 2557 October 6, 211981 (Natarajan \& Raaman 1983, 1984) as Coprinus lagopus.

Kerala: (Bhavani Devi 1995) as Coprinus lagopus.

Punjab: (Garcha \& Kalra 1977, Atri \& Kaur 2004) as Coprinus lagopus; Sangrur (231 m), Amargarh, growing in groups on mixed cattle dung, Amandeep Kaur, PUN 4819, June 21, 2008 (Amandeep et al. 2014).

80. Coprinopsis macrocephala (Berk.) Redhead, Vilgalys \& Moncalvo in Taxon 50(1): 229, 2001.

Tamil Nadu: Madras, Maduravoyal, Madras University Campus, growing in groups on ground, Herb. Mubl No. 2554, March 09, 1979 (Natarajan \& Raman 1983, 1984 as Coprinus macrocephalus.

Punjab: Jalandhar (233 m), Rahimpur, growing scattered on mixed cattle dung and straw residue heap, Amandeep Kaur, PUN 4820, July 30, 2010 (Amandeep et al. 2014).

81. Coprinopsis nivea (Pers.) Redhead, Vilgalys \& Moncalvo in Taxon 50(1): 229, 2001.

West Bengal (Mahju 1933) as Coprinus niveus.

West Bengal: Calcutta (Banerjee 1947) as Coprinus niveus.

Maharastra (Patil et al. 1995) as Coprinus niveus.

Kerala: Kottayam, growing solitary or in groups on cow dung during monsoon (Bhavani Devi 1995) as Coprinus niveus.

Punjab: growing on unspecified animal dung (Mahju 1933, Saini \& Atri 1995) as Coprinus niveus; Sangrur (231 m), Amargarh, growing in groups on buffalo dung, Amandeep Kaur, PUN 4821, June 21, 2008; Sangrur (231 m), Mahorana, growing in caespitose groups on buffalo dung, Amandeep Kaur, PUN 4822, June 21, 2008; Sangrur (231 m), Takhar, growing gregariously on buffalo dung, Amandeep Kaur, PUN 4823, June 26, 2008; Ludhiana (254 m), Nasrali, growing in groups on horse dung, Amandeep Kaur, PUN 4824, July 23, 2009; Sangrur (231 m), Chittanwala, growing in groups on buffalo dung flakes, Amandeep Kaur, PUN 4825, July 25, 2010 (Amandeep et al. 2014).

82. Coprinopsis patouillardii (Quél.) G. Moreno in Guía de los hongos de la Península Ibérica: 813, 2010.

According to Index Fungorum, the current valid name of Coprinus patouillardii is Coprinopsis patouillardii.

Punjab: Bir Bhadson (250 m), growing on cattle dung under Albizzia lebbek tree, Amanjeet Kaur, PUN 2954, September 09, 1999 (Atri \& Kaur 2004) as Coprinus patouillardii.

Karnataka: Heggala-Thora, Virajpet, Kodagu, growing on elephant dung, N.C. Karun, MUBSNCKKRSMF\#041, July 29, 2012 (Karun \& Sridhar 2015) as Coprinus patouillardii.

83. Coprinopsis pseudonivea (Bender \& Uljé) Redhead, Vilgalys \& Moncalvo in Taxon 50(1): 230, 2001.

Punjab: Sangrur (231 m), Langrian, growing in groups on cow dung, Amandeep Kaur, PUN 4062, June 21, 2008; Sangrur (231 m): Khurd, growing in groups on mixed cattle dung, Amandeep Kaur, PUN 4826, July 05, 2008; Tarn Taran (169 m): Kang, Khadoor Sahib, growing in a group of two on cow dung, Amandeep Kaur, PUN 4063, December 12, 2009 (Amandeep et al. 2014).

84. Coprinopsis radiata (Bolton: Fr.) Redhead, Vilgalys \& Moncalvo in Taxon 50(1): 230, 2001. According to MycoBank record, Coprinus fimetarius and Coprinus radiatus are synonyms of Coprinopsis radiata. Hence the names Coprinus fimetarius and Coprinus radiatus stand deleted and 
replaced by the name Coprinopsis radiata.

Jammu \& Kashmir: (Abraham 1991) as Coprinus radiatus.

Maharashtra: (Patil et al. 1995) as Coprinus fimetarius and Coprinus radiatus.

Kerala: growing solitary or in groups on dung heaps (Bhavani Devi 1995) as Coprinus fimetarius.

Punjab: growing on horse dung (Rea 1922, Mahju 1933) as Coprinus radiatus; Near Bhadson (250 m), Babulpur, growing on cattle dung, Amanjeet Kaur, PUN 2955, September 08, 1998 (Atri \& Kaur 2004) as Coprinus fimetarius; Patiala $(251 \mathrm{~m})$, Bhedpura, growing in a caespitose group on mixed cattle dung heap, Amandeep Kaur, PUN 4827, July 16, 2011 (Amandeep et al. 2014).

85. Coprinopsis radiata var. macrocarpa Atri, A. Kaur \& M. Kaur in Mycosphere 5(1): 15, 2014. Punjab: Sangrur $(231 \mathrm{~m})$, Bhasaur, growing in groups on buffalo dung, Amandeep Kaur, PUN 4828, September 15, 2007; Sangrur (231 m), Langrian, growing in groups on mixed cattle dung, Amandeep Kaur, PUN 4829, June 21, 2008; Patiala (251 m), Chhat Bir, growing in groups on mixed cattle dung heap, Amandeep Kaur, PUN 4830, June 30, 2008; Sangrur (231 m), Sandaur, growing scattered on mixed cattle dung, Amandeep Kaur, PUN 4831, September 29, 2008 (Amandeep et al. 2014).

86. Coprinopsis scobicola (P.D. Orton) Redhead, Vilgalys \& Moncalvo in Taxon 50(1): 231, 2001.

Punjab: Sangrur (231 m), Meemsa, growing solitary on sheep dung, Amandeep Kaur, PUN 4832, July 25, 2010 (Amandeep et al. 2014).

87. Coprinopsis vermiculifer (Joss.: Dennis) Redhead, Vilgalys \& Moncalvo in Taxon 50(1): 232, 2001.

Punjab: Hoshiarpur (295 m), Garhshankar, growing in a group on buffalo dung, Amandeep Kaur, PUN 4833, July 5, 2011(Amandeep et al. 2014).

(xx) Genus: Panaeolus (Fr.) Quél. in Mémoires de la Société d'Émulation de Montbéliard 5: 151, 1872. Type Species: Panaeolus papilionaceus (Bull.) Quél. (1872)

88. Panaeolus acuminatus Quél. in Hyménomycètes, Fasc. Suppl. (Alençon): 621, 1874.

Kerala: Silent Valley, TBGRI Campus, growing scattered on elephant dung (Vrinda et al. 1999).

Punjab: Bathinda $(211 \mathrm{~m})$, Lehra Mohabbat, growing gregariously in cattle pasture, Amandeep Kaur, PUN 4030, August 02, 2009 (Kaur et al. 2014c).

89. Panaeolus africanus Oláh in Rev. Mycol. Mem. Ser. 10: 125, 1969.

Tamil Nadu: Madras, Guindy, Children's Park, growing in groups on elephant dung, Herb. MUBL No. 2574, December 12, 1978 (Natarajan \& Raaman 1983, 1984).

90. Panaeolus africanus var. diversistipus Amandeep Kaur, NS Atri \& Munruchi Kaur in Mycosphere 4(3): 620, 2013.

Punjab: Hoshiarpur (295 m), Jejon Duaba, growing solitary on mixed cattle dung heap, Amandeep Kaur, PUN 4342, July 5, 2011(Amandeep et al. 2013a).

91. Panaeolus alcis M.M. Moser in Mycologia 76 (3): 551, 1984.

Punjab: Moga $(217 \mathrm{~m})$, Chak Fatehpur, growing scattered or in caespitose groups on buffalo dung, Amandeep Kaur, PUN 4359, June 28, 2011(Kaur et al. 2014c).

92. Panaeolus annulatus Natarajan \& Raman in Bibliotheca Mycologica 89: 52, 1983.

Tamil Nadu: Madras, Guindy, Children's Park, growing on elephant dung, Herb. MUBL No. 2579, December 08, 1980 (Natarajan \& Raaman 1983, 1984).

93. Panaeolus antillarum (Fr.) Dennis in Kew Bulletin 15 (1):124, 1961.

Tamil Nadu: Madras, Guindy, Children's Park, growing in groups on elephant dung, August 23, 1978, 
December 12, 1978. Herb. MUBL No. 2575, 2576 (Natarajan and Raaman, 1983, 1984).

Kerala: Malappuram, Ramapuram, growing on elephant dung, August 16, 2002, Nisha NVS109; August 18, 2002, Nisha NVS109b; Thrissur, Guruvayur, October 28, 2002, Nisha NVS109c; March 09, 2003, Nisha NVS109d; April 11, 2003, Nisha NVS109e (Manimohan et al. 2007); (Mohanan 2011).

Punjab: Sangrur (231 m), Ratolan, growing solitary on mixed cattle dung, Amandeep Kaur, PUN 4225, September 28, 2008 (Kaur et al. 2014c).

94. Panaeolus ater (J.E. Lange) Kühner \& Romagn. ex Bon in Documents Mycologiques 16 (61): 46, 1985.

Kerala: Kottayam, Ranni, growing solitary or scattered on the droppings of herbivorous animals (Bhavani Devi 1995).

Punjab: Fatehgarh Sahib (228 m), Nogwaan, growing scattered on cattle dung, Amandeep Kaur, PUN 4032, August 21, 2009; Hoshiarpur (295 m), Chak Sadhu, growing in groups on buffalo dung, Narinderjit Kaur, PUN 4704, July 22, 2011(Kaur et al. 2014c).

95. Panaeolus cyanescens (Berk. \& Br.) Sacc. in Sylloge Fungorum 5: 1123, 1887.

Bose (1920) described the species for the first time from India as Panaeolus cyanescens. Later Ghosh et al. (1967) described it as Copelandia cyanescens. Manjula (1983) put the name Panaeolus cyanescens in the excluded list and described Copelandia cyanescens as the valid name. Natarajan \& Raaman (1983, 1984) and Manimohan et al. (2007) described it under the name Copelandia cyanescens. But MycoBank record shows that Panaeolus cyanescens is the legitimate name for the species and Copelandia cyanescens is an obligate synonym of this name. Hence, the name Copelandia cyanescens stands replaced in the Indian record by Panaeolus cyanescens.

West Bengal: on dung heaps (Bose 1920).

Uttar Pradesh: Lucknow (Ghosh et al. 1967) as Copelandia cyanescens.

Tamil Nadu: Madras, Guindy, growing in groups on elephant dung, Herb. MUBL No. 2570, November 02, 1978 (Natarajan \& Raman 1983, 1984) as Copelandia cyanescens.

Jammu and Kashmir: Abraham (1991); Lakhanpal (1993).

Kerala: Malappuram, Angadipuram, growing on elephant dung, Nisha NVS107, August 12, 2002; Thrissur, Guruvayur, Nisha NVS128, September 09, 2002 (Manimohan et al. 2007) as Copelandia cyanescens.

Punjab: Fatehgarh Sahib (228 m), Sirhind, along G.T. Road, growing in groups on mixed dung and humicolous soil under Eucalyptus citridora tree, Amanjeet Kaur, PUN 2708, September 16, 1995; Fatehgarh Sahib (228 m), Sirhind, growing in caespitose groups on cattle manure in the field of Allium sativum crop, Amanjeet Kaur, PUN 2712, November 17, 1995; Fatehgarh Sahib (228 m), Sirhind, growing in groups on cattle dung, Amanjeet Kaur, PUN 2707, November 17, 1995; Fatehgarh Sahib (228 m), Sirhind, growing in groups on cattle manured soil, Amanjeet Kaur, PUN 2711, November 27, 1995; Patiala (250 m), Bir Bhunerheri, growing scattered on cattle manured soil near Parthenium grass, Amanjeet Kaur, PUN 2710, March 07, 1998; Fatehgarh Sahib (228 m), Bassi, growing scattered on mixed dung, Amanjeet Kaur, PUN 2713, September 14, 1998; Fatehgarh Sahib (228 m), Sirhind, growing in groups on cattle dung manured soil in Allium sativum field, Amanjeet Kaur, PUN 2709, November 28, 1998; Sangrur (251 m), Malak Majra, growing gregariously on buffalo dung, Amandeep Kaur, PUN 4355, June 23, 2008; Patiala (251 m), Dakala, Dashmesh Nagar, growing gregariously on cow dung, Amandeep Kaur, PUN 4077, June 25, 2008; Patiala (251 m), Chhat Bir, growing in caespitose clusters on buffalo dung, Amandeep Kaur, PUN 4028, June 30, 2008; Patiala (251 m), Chhat Bir, growing gregariously on buffalo dung, Amandeep Kaur, PUN 4078, June 30, 2008; Hoshiarpur (295 m), Simbli, growing scattered on mixed cattle dung, Harwinder Kaur, PUN 4361, July 19, 2008; Ludhiana $(254 \mathrm{~m})$, growing in groups on cattle dung, Baljit Kaur, PUN 3922, September 03, 2008; Ludhiana $(254 \mathrm{~m})$, Nasrali, growing scattered in groups on mixed cattle dung, Amandeep Kaur, PUN 4079, July 23, 2009; Mohali (31 6m), Bhajauli, growing scattered on cow dung heap, Amandeep Kaur, PUN 4031, August 21, 2009; Ropar (394 m), Kuraali, growing solitary on mixed cattle dung, 
Amandeep Kaur, PUN 4033, August 21, 2009; Sangrur (251 m): Jaatimajra, growing gregariously on horse dung, Amandeep Kaur, PUN 4080, September 03, 2009; Patiala (251 m), Chhat Bir, growing in groups on elephant dung, Amandeep Kaur, PUN 4353, July 10, 2010; Patiala (251 m), Chhat Bir, growing scattered on elephant dung, Amandeep Kaur, PUN 4354, July 10, 2010; Ropar (394 m), near Haveli, growing in groups on buffalo dung, Arpana Lamba, PUN 4296, July 16, 2010; Ropar (394 m), growing in groups on buffalo dung, Arpana Lamba, PUN 4297, July 25, 2010 (Kaur et al. 2014c).

96. Panaeolus cyanoannulatus Atri, M. Kaur \& A. Kaur in JNBR 3(2): 126, 2014.

Punjab: Hoshiarpur (295 m), Jeewanpur Jattan, found growing in a group on a mixed cow and horse dung heap in a pasture, Amandeep Kaur, PUN 4223, July 18, 2008 (Kaur et al. 2014a).

97. Panaeolus fimicola (Pers.) Gillet in Hyménomycètes (Alençon): 621, 1878.

Karnataka: Makutta Reserve forest, Virajpet, Kodagu, growing on elephant dung, N.C. Karun, MUBSNCKKRSMF \# 042, August 20, 2012 (Karun \& Sridhar 2015).

98. Panaeolus lepus-stercus Atri, M. Kaur \& A. Kaur in JNBR 3(2): 129, 2014.

Punjab: Pathankot (309 m), Sheep and Rabbit Breeding Farm Dalla Dhar, growing scattered on rabbit pellets, Amandeep Kaur, PUN 4340, September 01, 2011(Kaur et al. 2014a).

99. Panaeolus papilionaceus (Bull.) Quél. In Mém. Soc. Émul. Montbéliard, Sér. 2,5: 152 [122 repr.], 1872.

Tamil Nadu: Kodaikanal, Konalaru, Pine Regeneration Area, growing solitary on ground, August 13, 1978. Herb. MUBL No. 2578 (Natarajan \& Raaman 1983, 1984) as Panaeolus sphinctrinus.

Punjab: Hoshiarpur (295 m), Mahilpur, growing gregariously on horse dung in a pasture, Munruchi Kaur and Amandeep Kaur, PUN 4224, July 18, 2008; Bathinda (211 m), Lehra Mohabbat, growing gregariously in caespitose groups on cow dung, Amandeep Kaur and Harwinder Kaur, PUN 4029, August 02, 2009 (Kaur et al. 2014c) as Panaeolus sphinctrinus; Sangrur (231 m), Dugni, growing in groups on buffalo dung among grasses along roadside, Amandeep Kaur, PUN 4360, June 27, 2011(Kaur et al. 2014c) as Panaeolus papilionaceus var. parvisporus.

100. Panaeolus rickenii Hora in Trans. Brit. Mycol. Soc. 43: 454, 1960.

Kerala: Thrissur, Guruvayur, growing on elephant dung, Nisha NVS129, September 09, 2002; Malappuram, Ramapuram, growing on elephant dung, Nisha NVS133; November 11, 2002, (Manimohan et al. 2007).

101. Panaeolus semiovatus (Sowerby) S. Lundell \& Nannf. in Fungi Exsiccati Suecici Fasc. 11-12 (537), 1938.

Bhavani Devi (1995) reported this species as Anellaria semiovata. MycoBank record refers this name as a synonym of Panaeolus semiovatus. Now, Anellaria semiovata stands deleted from Indian list and replaced by Panaeolus semiovatus which is the legitimate name.

Kerala: Konni, Pathanamthitta, growing solitary or scattered on elephant dung (Bhavani Devi 1995 as Anellaria semiovata).

102. Panaeolus solidipes (Peck) Sacc. in Sylloge Fungorum 5: 1123, 1887.

Kerala: Trivandrum, Vellayani, Agricultural College Campus, growing solitary or scattered on manured ground (Bhavani Devi 1995).

Punjab: Sangrur $(231 \mathrm{~m})$, Upoki, growing solitary on horse dung, Amandeep Kaur, PUN 4034, September 4, 2009 (Kaur et al. 2014c).

103. Panaeolus speciosus var. pilocystidiosus Amandeep Kaur, NS Atri \& Munruchi Kaur in Mycosphere 4(3): 622, 2013. 
Punjab: Barnala (228m), Rarh, growing scattered on cattle dung, Amandeep Kaur, PUN 4081, June 26, 2008 (Amandeep et al. 2013a).

104. Panaeolus subbalteatus (Berk. \& Br.) Sacc. in Sylloge Fungorum 5: 1124, 1887.

Tamil Nadu: Madras, Guindy, Deer Park, growing in groups on elephant dung, November 02, 1979, Herb. MUBL No. 2577 (Natarajan \& Raaman 1983, 1984).

Jammu \& Kashmir: Lakhanpal (1986); Abraham (1991).

Punjab: Barnala (228 m), Wazeedake, growing in groups on buffalo dung among grasses, Amandeep Kaur, PUN 4228, July 31, 2009; Bathinda (211m), Nandgarh, growing scattered on buffalo dung, Amandeep Kaur, PUN 4227, August 01, 2009; Ropar (394 m), Padiala, growing in groups on a mixed cattle dung heap, Amandeep Kaur, PUN 4229, August 21, 2009; Ropar (394 m): Kiratpur Sahib, growing in groups on mixed cattle dung, Harwinder Kaur, PUN 4770, July 13, 2012 (Kaur et al. 2014c).

105. Panaeolus tropicalis Oláh in Rev. Mycol. 4: 289, 1969.

Punjab: Patiala $(251 \mathrm{~m})$, Nainakut, growing in groups on mixed cattle dung, Amandeep Kaur, PUN 4076, June 16, 2008; Patiala (251 m), Bhunerheri, growing in groups on mixed cattle dung, Amandeep Kaur, PUN 4346, June 16, 2008; Hoshiarpur (295 m), Kot Fatuhi, growing solitary on mixed cattle dung among grassy litter, Narinderjit Kaur, PUN 4341, August 18, 2011(Kaur et al. 2014c).

106. Panaeolus venezolanus Guzmán in Mycotaxon 7 (2): 221, 1978.

Punjab: Faridkot $(196 \mathrm{~m})$, Panjgraean, growing in a group on cattle dung and wheat straw mixture heap, Amandeep Kaur, PUN 4834, August 19, 2011(Kaur et al. 2014c).

(xxi) Genus: Parasola Redhead, Vilgalys \& Hopple in Taxon 50(1): 235, 2001.

Type species: Parasola plicatilis (Curtis) Redhead, Vilgalys \& Hopple

The genus Parasola was established by Readhead et al. (2001). Erstwhile Coprinus species having smooth pileus and stipe surfaces, non-deliquescent lamellae and hymeniform pileus cuticle have been placed under the newly established genus Parasola Redhead, Vilgalys \& Hopple.

107. Parasola conopilus (Fr.) Örstadius \& E. Larss. In Mycol. Res.112(10): 1180, 2008.

Kerala: growing scattered on elephant dung (Vrinda et al. 1999) as Psathyrella conopilus.

Punjab: Sangrur $(231 \mathrm{~m})$, Bhudan, growing solitary on buffalo dung and vegetable waste heap, Amandeep Kaur, PUN 4073, January 20, 2008.

108. Parasola plicatilis (Curtis) Redhead, Vilgalys \& Hopple in Taxon 50(1):235, 2001.

Watling and Gregory (1980) recorded Coprinus plicatilis which has now been reclassified as Parasola plicatilis which is a valid name. Hence the name Coprinus plicatilis stands deleted from the Indian record.

Tamil Nadu: Madras, Guindy, Children's park, growing in groups on ground, Herb. Mubl No. 2559, September 24, 1979 (Natarajan \& Raaman 1983, 1984).

Kerala: growing solitary in grassland and gardens (Bhavani Devi 1995).

Jammu \& Kashmir: Dachigan Reserve (Watling \& Gregory 1980) as Coprinus plicatilis; Abraham (1991).

Punjab: Atri \& Kaur (2004); Kapurthala (224 m), growing scattered on manured soil, Jyoti Mann and Amandeep Kaur, PUN 4059, May 15, 2007.

(xxii) Genus: Psathyrella Fr.: Quél. in Mém. Soc. Émul. Montbéliard 5: 152, 1872.

Type species: Psathyrella gracilis (Fr.) Quél.

109. Psathyrella castaneifolia (Murrill) A.H. Sm., Mem. N. Y. bot. Gdn 24: 33, 1972.

Punjab: Sangrur $(231 \mathrm{~m})$, Sikanderpura, growing in groups on mixed cattle dung, Amandeep Kaur, 
PUN 4358, June 29, 2011; Patiala (251 m), Ghanaur, growing solitary on buffalo dung, Amandeep Kaur, PUN 4357, July 19, 2011(Kaur et al. 2014c) as Panaeolus castaneifolius.

110. Psathyrella fimicola NS Atri, Munruchi Kaur and Amandeep Kaur in JNBR 2(3): 276, 2013. Punjab: Patiala (251m), Harigarh, growing in group on horse dung, Amandeep Kaur, PUN 4317, June 18, 2011(Kaur et al. 2013b).

111. Psathyrella flocculosa (Earle) A.H. Smith in Mem. N. Y. Bot. Gdn. 24: 181, 1972.

Punjab: Sangrur $(231 \mathrm{~m})$, Naushehra, growing gregariously on mixed cattle dung heap, Amandeep Kaur, PUN 4074, July 07, 2007.

112. Psathyrella kauffmanii var. kauffmanii Smith in Mem. N. Y. Bot. Gdn. 24: 69, 1972.

Punjab: Moga $(217 \mathrm{~m})$, Loahgarh, growing in caespitose clusters on buffalo dung heap under Azadirachta indica tree, Amandeep Kaur, PUN 4318, July 28, 2009.

113. Psathyrella sphaerocystis Orton in Notes Roy. Bot. Gard. Edinburgh 26: 57. 1964.

Punjab: Sangrur $(231 \mathrm{~m})$, Balamgarh, growing in caespitose cluster on mixed cattle dung heap, Amandeep Kaur, PUN 4075, July 30, 2009.

114. Psathyrella vanhermanii Smith in Mem. N. Y. Bot. Gdn. 24: 79, 1972.

Punjab: Mohali (316 m), Parol, growing in groups on buffalo dung, Amandeep Kaur, PUN 4316, July 14, 2007; Ludhiana (254 m): Issru, growing in groups on buffalo dung, Amandeep Kaur, PUN 4315, June 17, 2008.

IX. Family: Strophariaceae Singer \& Smith in Mycologia 38: 503, 1946.

Type genus: Stropharia (Fr.) Quél.

(xxiii) Genus: Agrocybe Fayod in Annales des Sciences Naturelles Series VII, 9: 358, 1889.

Type species: Agrocybe praecox (Pers.: Fr.) Fayod

115. Agrocybe guruvayoorensis K. A. Thomas \& Manim. in Mycotaxon 86: 330, 2003.

Kerala: Thrissur, Guruvayur, growing on elephant dung, A. Thomas T316, July 14, 1999, A. Thomas T316b, July 16, 1999, A. Thomas T316c, July 21, 1999, A. Thomas T316d, October 18, 1999 (Thomas \& Manimohan 2003, Manimohan et al. 2007).

116. Agrocybe microspora Singer in Sydowia 30(1-6): 205, 1977.

Punjab: Patiala (251 m): Bahadurgarh, growing in groups on manured soil in a cattle pasture, Munruchi Kaur and Yadwinder Singh, PUN 4835, May 28, 2008 (Kaur et al. 2014d).

117. Agrocybe pediades (Fr.) Fayod in Annales des Sciences Naturelles, Series 7,9: 358, 1889.

Kerala: ( Mohanan 2011)

Uttar Pradesh: Saharanpur (Hennings 1901).

Chandigarh (321 m): growing on dung (Rawla et al. 1982, Saini \& Atri 1995) as Agrocybe semiorbicularis.

Punjab; Sangrur (231m): Kelon, growing in groups on mixed cattle dung, Amandeep Kaur, PUN 4226, August 14, 2008 (Kaur et al. 2014d).

(xxiv) Genus: Protostropharia Redhead, Moncalvo \& Vilgalys in Index Fungorum 15:2, 2013.

Type species: Protostropharia semiglobata (Batsch) Redhead, Moncalvo \& Vilgalys

118. Protostropharia. semiglobata (Batsch) Redhead, Moncalvo \& Vilgalys in Index Fungorum 15: 2 , 2013. 
Assam: Khasi Hills (Berkeley 1852) as Agaricus semiglobata.

Punjab: growing on camel dung (Rea 1922, Ginai 1936, Saini \& Atri 1995) as Stropharia semiglobata. Jammu and Kashmir: Gulmarg (Watling \& Gregory 1980) as Stropharia semiglobata.

Tamil Nadu: Nilgiris, Ootacamud, Naduvattam, growing in groups on dung, Herb. Mubl No. 2604, September 08, 1978 (Natarajan \& Raaman 1983, 1984) as Stropharia semiglobata.

Kerala: growing solitary or gregarious on dung, or manured soil (Bhavani Devi 1995); (Mohanan 2011 as Stropharia semiglobata).

119. Protostropharia semiglobata var. punjabensis Amandeep Kaur, NS Atri and Munruchi Kaur in Kavaka 41: 11, 2013.

Punjab: Pathankot (309 m), Berkula, growing solitary on cow dung in an open pasture, Munruchi Kaur and Amandeep Kaur, PUN 4840, September 02, 2011(Kaur et al. 2013c).

(xxv) Genus: Psilocybe (Fr.) P. Kumm. in Der Führer in die Pilzkunde 1: 21, 71, 1871.

Type species: Psilocybe montana (Pers.) P. Kumm.

120. Psilocybe argentina (Speg.) Singer in Nova Hedwigia Beih. 29: 241, 1969.

Tamil Nadu: Nilgiris, Ootacamud, Santinella, growing in groups on cow dung, Herb. MUBL No. 2627, November 06, 1979 (Natarajan \& Raman 1983, 1984).

Kerala: Idukki, Munnar, Thomas T150b, August 28, 1997 (Thomas \& Manimohan 2002); (Mohanan 2011).

121. Psilocybe aztecorum R. Heim in Revue Mycol., Paris 22: 78, 1978.

Punjab: Patiala (251 m), Wazeedpur, growing solitary on buffalo dung, Amandeep Kaur, PUN 4837, July 16, 2011; Pathankot (309 m): Shahpur Kandi, growing in group on buffalo dung, Munruchi Kaur and Amandeep Kaur, PUN 4838, September 01, 2011.

122. Psilocybe bonetii Guzmán in Anais da Escola nac. Cienc. biol., Méx. 17(1-4): 9, 1970.

Tamil Nadu: Nilgiris, Ootacamud, Santinella, growing in groups on cow dung, Herb. MUBL No. 2621, November 06, 1979 (Natarajan \& Raaman 1983, 1984) as Psilocybe aztecorum var. bonetii.

Punjab: Hoshiarpur, Badowan (295 m), growing scattered on a buffalo dung flake, Munruchi Kaur and Amandeep Kaur, PUN 4836, July 18, 2008.

123. Psilocybe coprophila (Bull.) P. Kumm. in Der Führer in die Pilzkunde: 71, 1871.

Jammu \& Kashmir: Watling \& Gregory (1980).

Tamil Nadu: Kodaikanal, Konalaru, growing solitary and in groups on dung, Herb. MUBL No. 2626, August 13, 1978 (Natarajan \& Raaman 1983, 1984).

Kerala: growing gregariously or scattered on elephant dung (Bhavani Devi, 1995); Idukki, Munnar, growing on elephant dung A. Thomas T149, August 28, 1997 (Thomas \& Manimohan 2002, Manimohan et al. 2007).

Karnataka: Shola Forest of Heggala-Thora, Virajpet, Kodagu, growing on elephant dung, N.C. Karun, MUBSNCKKRSMF \# 044, July 29, 2012 (Karun \& Sridhar, 2015).

124. Psilocybe cubensis (Earle) Singer in Sydowia 2: 37, 1948.

Tamil Nadu: Madras, Guindy, Deer Park, growing solitary on elephant dung manure, Herb. MUBL No. 2613, November 02, 1978 (Natarajan \& Raaman 1983, 1984).

Kerala: Idukki, Munnar, growing on soil with heavy traffic of cattle, Thomas T156, August 29, 1997, Thomas T156b, August 30, 1997, Thomas T156c, August 30, 1997 (Thomas \& Manimohan 2002).

125. Psilocybe fimetaria (P.D. Orton) Watling in Lloydia 30 (2): 150, 1967.

Karnataka: Shola Forest, Heggala-Thora, Virajpet, Kodagu, growing on elephant dung, N.C. Karun, MUBSNCKKRSMF \# 045, September 07, 2012 (Karun \& Sridhar 2015). 
126. Psilocybe gigaspora Natarajan \& Raaman in Bibliotheca Mycologica 89: 100, 1983.

Tamil Nadu: Nilgiris, Ootacamud, Santinella, growing in groups on cow dung, Herb. MUBL No. 2611, November 06, 1979 (Natarajan \& Raaman 1983, 1984).

127. Psilocybe inquilina (Fr.) Bres. in Iconographia Mycologica 18: 863, 1931.

Kerala: (Mohanan 2011).

128. Psilocybe merdaria (Fr.) Ricken in Die Blätterpilze: 251, 1912.

Maharashtra: Bombay, Poona, on dung (Massee 1901) as Stropharia merdaria.

129. Psilocybe pegleriana Guzmán in Doc. Mycol. 29 (116): 43, 2000.

Kerala: Thrissur, Guruvayur, growing on elephant dung, A. Thomas T311, June 11, 1999, A. Thomas T311b, June 17, 1999, A. Thomas T311c, July 14, 1999; Palakkad, Nelliyampathy, A. Thomas T311d, August 30, 1999; Kasaragod, Adhoor, A. Thomas T311e, September 16, 2000 (Manimohan et al. 2007); (Mohanan 2011).

130. Psilocybe semilanceata (Fr.) P. Kumm. in Der Führer in die Pilzkunde p. 71, 1871.

Maharashtra: Patil et al. (1995).

Punjab: Patiala $(251 \mathrm{~m})$, Bhunerheri, growing in groups on mixed cattle dung heap in a pasture, Amandeep Kaur, PUN 4839, June 16, 2008.

131. Psilocybe subaeruginascens Höhn in Sitzungsber. Kaiserl. Akad. Wiss., Math-Naturwiss. KI., AbL I, 123 (1): 78, 1914.

Kerala: Calicut, Vellarimala, growing on elephant dung, K. A. Thomas T170, September 19, 1999; K. A. Thomas T170b, September 20, 1997, K. A. Thomas T170c, November 08, 1999 (Thomas \& Manimohan 2002, Manimohan et al. 2007).

132. Psilocybe subcubensis Guzmán in Mycotaxon 7: 248, 1978.

Kerala: Wayanad, Muthanga, growing on elephant dung, K. A. Thomas T76, June 17, 1997; K. A. Thomas T76b, June 25, 1997; K. A. Thomas T76c, July 04, 1997; K. A. Thomas T76d, August 19, 1997; K. A. Thomas T76e, October 01, 1997; K. A. Thomas T76f, October 26, 1997; K. A. Thomas T76g, May 25, 1999; K. A. Thomas T76h, July 21, 1999; Malappuram, Nilambur, Nisha NVS 142, June 28, 2003 (Thomas \& Manimohan 2002, Manimohan et al. 2007); (Mohanan 2011).

(xxvi) Genus: Stropharia (Fr.) Quél. in Mém. Soc. Émul. Montbéliard 5: 141, 1872.

Type species: Stropharia aeruginosa (Curtis) Quél.

133. Stropharia bicolor Pegler in Kew. Bull. Addit. Ser. 6: 463, 1977.

Kerala: Idukki, Munnar, growing on elephant dung, A. Thomas T152, August 29, 1997; Palakkad, Nelliyampathy, A. Thomas T152 b, August 29, 1999, A. Thomas T152 c, August 30, 1999 (Manimohan et al. 2007).

134. Stropharia rugosoannulata Farl.: Murrill in Mycologia 14: 139, 1922.

Kerala: Wayanad, Muthanga, growing on elephant dung, A. Thomas T75, June 17, 1997, A. Thomas T75b, June 25, 1997, A. Thomas T75c, July 04, 1997, A. Thomas T75e, July 21, 1999, A. Thomas T75f, July 25, 1999; Palakkad, Nelliyampathy, A. Thomas T75d, August 15, 1997 (Manimohan et al. 2007).

X. Family: Tricholomataceae R. Heim: Pouzar in Ceská Mykol. 37(3): 174, 1983.

Type genus: Tricholoma (Fr.) Staude 
(xxvii) Genus: Macrocybe Pegler \& Lodge in Mycologia 90: 496, 1998.

Type species: Macrocybe titans (H.E. Bigelow \& Kimbr.) Pegler, Lodge \& Nakasone

135. Macrocybe gigantea (Massee) Pegler \& Lodge in Mycologia 90: 497, 1998.

Kerala: Thrissur, Guruvayur, growing on elephant dung, P. Manimohan M750, May 27, 1999, P. Manimohan M750a, June 17, 1999 (Manimohan et al. 2007).

Table 1 List showing excluded and replaced names of coprophilous mushrooms in India

\begin{tabular}{|c|c|}
\hline Excluded names & Current names \\
\hline Coprinaceae & Agaricaceae \\
\hline Agrocybe semiorbicularis & Agrocybe pediades \\
\hline Anellaria semiovata & Panaeolus semiovatus \\
\hline Bolbitius glatfelteri & Mycena glatfelteri \\
\hline Bolbitius vitellinus & Bolbitius titubans \\
\hline Conocybe albipes & Conocybe apala \\
\hline Conocybe magnicapitata & Conocybe juniana \\
\hline Conocybe plumbeitincta & Pholiotina plumbeitincta \\
\hline Copelandia cyanescens & Panaeolus cyanescens \\
\hline Coprinus cinerea & Coprinopsis cinerea \\
\hline Coprinus cordisporus & Coprinopsis cordispora \\
\hline Coprinus ephemerus & Coprinellus ephemerus \\
\hline Coprinus fimbriatus & Coprinellus fimbriatus \\
\hline Coprinus fimetarius & Coprinopsis radiata \\
\hline Coprinus lagopides & Coprinopsis lagopides \\
\hline Coprinus lagopus & Coprinopsis lagopus \\
\hline Coprinus macrocephalus & Coprinopsis macrocephala \\
\hline Coprinus micaceus & Coprinellus micaceus \\
\hline Coprinus niveus & Coprinopsis nivea \\
\hline Coprinus patouillardii & Coprinopsis patouillardii \\
\hline Coprinus plicatilis & Parasola plicatilis \\
\hline Coprinus radiatus & Coprinopsis radiata \\
\hline Coprinus truncorum & Coprinellus truncorum \\
\hline Galera antipus & Conocybe antipus \\
\hline Lepiota cepistipes & Leucocoprinus cepistipes \\
\hline Lepiota humei & Chlorophyllum humei \\
\hline Lepiota meleagris & Leucoagaricus meleagris \\
\hline Lepiota sordescens & Leucocoprinus cepistipes \\
\hline Leucoagaricus naucinus & Leucoagaricus leucothites \\
\hline Leucocoprinus cretatus & Leucocoprinus cretaceus \\
\hline Macrolepiota rhacodes & Chlorophyllum rhacodes \\
\hline Panaeolina rhombisperma & Crucispora rhombisperma \\
\hline Panaeolus castaneifolius & Psathyrella castaneifolia \\
\hline Panaeolus papilionaceus var. parvisporus & Panaeolus papilionaceus \\
\hline Panaeolus sphinctrinus & Panaeolus papilionaceus \\
\hline Psathyrella conopilus & Parasola conopilus \\
\hline Psilocybe aztecorum var. aztecorum & Psilocybe aztecorum \\
\hline Psilocybe aztecorum var. bonetii & Psilocybe bonetii \\
\hline Stropharia merdaria & Psilocybe merdaria \\
\hline Stropharia semiglobata & Protostropharia semiglobata \\
\hline Volvariella earlei & Volvopluteus earlei \\
\hline Volvariella gloiocephala & Volvopluteus gloiocephalus \\
\hline Volvariella speciosa & Volvopluteus gloiocephalus \\
\hline
\end{tabular}

\section{Discussion}

India exhibits marked variations in climate, vegetation and livestock population. The extent of available information about coprophilous mushrooms from India in general is meager and there is much scope for exploratory work on these mushrooms. In the present work, systematic exploration on coprophilous mushrooms was undertaken in Punjab state with a focus on taxonomy, diversity and 
ecological aspects of coprophilous mushrooms. In this cheklist all the coprophilous agarics recorded previously from different parts of India to date, including those in the investigation of Punjab state, have been listed. The work done has vast scope for further extension as 135 taxa have been documented from 13 states and 2 union territories. The knowledge generated by the work is of immense utility as it is key to revealing the diversity and ecology of coprophilous agarics. However, the list covers only a part of the actual diversity of these mushrooms in India as most of the relevant original information is literature-based and many of the papers bear only limited information on habit and habitat. Moreover, studies on the coprophilous agarics have been inadequate in the country and therefore there still maybe many undescribed coprophilous mushrooms which may have been missed due to their fragility and tendency to disappear quickly in addition to the many unsurveyed states. The work demonstrates that dung is a significant substrate which serves as a favorable niche for the growth of a variety of mushrooms. Coprophilous mushrooms must be conserved as they play a significant role in the sustenance of ecological balance on the earth.

\section{Acknowledgement}

The authors wish to thank The Head, Department of Botany, Punjabi University, Patiala for providing laboratory facilities. The grant-in-aid under SAP-III programme by University Grants Commission, New Delhi to the Department of Botany, Punjabi University, Patiala, Punjab is also acknowledged.

\section{References}

123India.com - http://www.123india.com (accessed on March 31, 2015).

Abraham SP 1991 - Kashmir fungal flora- An Overview. In: Indian Mushrooms (ed Dr. MC Nair). Kerala Agricultural University, Velenikkara, pp 13-24.

Agha ZD 1978 - Review of mushroom growing in Jammu and Kashmir. Indian Mushroom Science 1, 49-54.

Amandeep K, Atri NS, Munruchi K. 2013a - Two new coprophilous varieties of Panaeolus (Psathyrellaceae, Agaricales) from Punjab, India. Mycosphere 4(3), 616-625, Doi 10.5943/mycosphere/4/3/13.

Amandeep K, Atri NS, Munruchi K. 2013b - Diversity of species of the genus Bolbitius (Bolbitiaceae, Agaricales) collected on dung from Punjab, India. Mycosphere 4(6), 1053-1064, Doi 10.5943/mycosphere/4/6/3.

Amandeep K, Atri NS, Munruchi K. 2014 - Taxonomic study on coprophilous species of Coprinopsis (Psathyrellaceae, Agaricales) from Punjab, India. Mycosphere 5(1), 1-25, Doi 10.5943/mycosphere/5/1/1

Amandeep K, Atri NS, Munruchi K. 2015a - Diversity of species of the genus Conocybe (Bolbitiaceae, Agaricales) collected on dung from Punjab, India. Mycosphere 6(1), 19-42, Doi 10.5943/mycosphere/6/1/4.

Amandeep K, Atri NS, Munruchi K. 2015b - Taxonomic study on the coprophilous mushrooms from Punjab, India: new records of family Agaricaceae. Current Research in Environmental \& Applied Mycology 5(1), 27-45, Doi 10.5943/cream/5/1/5

Atri NS, Kaur A, Kaur M. 2009 - Three new records of coprophilous mushrooms of family Bolbitiaceae from India. Mushroom Research 18 (2), 51-56.

Atri NS, Kaur A, Kour H. 2005 - Systematics and Sociobiology of Termitophilous mushrooms from Punjab. In: Fungi- Diversity and Conservation in India (eds JS Dargan, NS Atri, GS Dhingra). Bishen Singh Mahendra Pal Singh, Dehra Dun, UA (India), pp 159-182.

Atri NS, Kaur A. 2004 - Mushroom flora of Patiala- The genus Coprinus Pers. ex Gray. In: Plant Diversity in India (eds JS Dargan, TA Sarma). Bishen Singh Mahendra pal Singh, Dehra Dun, UA (India), pp 427-448.

Atri NS, Kaur M, Kaur A. 2012 - Taxonomic studies on some coprophilous species of Conocybe from India. Mushroom Research 21(2), 103-109. 
Atri NS, Kumari B, Upadhyay RC. 2014 - Taxonomy, Sociobiology, Nutritional and Nutraceutical Potential of Termitophilous and Lepiotoid Mushrooms from North West India. Proceedings of the 8th International Conference on Mushroom Biology and Mushroom Products, ICMBMP8, 19-22 November, 2014, New Delhi, India, pp 479-489.

Atri NS, Saini SS, Kaur A. 2000 - Taxonomical studies on Agarics from Punjab - The genus Lepiota (Pers. ex. Fr.) Gray. Mushroom Research 9(2), 71-78.

Atri NS, Saini SS, Kaur G. 1992 - Taxonomic studies on some members of family Bolbitiaceae Sing. from Punjab. Journal of the Indian Botanical Society 71(1-2), 87-89.

Atri NS, Saini SS, Kaur G. 1996 - Three species of agarics from Patiala. Mushroom Research 5(2), 77 80.

Balfour E 1976 -Encyclopaedia Asiatica: Comprising Indian Subcontinent, Eastern and Southern Asia. Cosmo Publications. ISBN 81-7020-325-2

Banerjee SN 1947 - Fungal flora of Calcutta and suburbs I. Bulletin of the Botanical Society of Bengal $1,37-54$.

Berkeley MJ 1851 - Decades 32, 33, Sikkim Himalayan Fungi. Hooker Journal of Botany 3, 39-49.

Berkeley MJ 1852 - Sikkim and Khassya Fungi. Hooker Journal of Botany 4,130-142.

Bhattacharya B, Baruah HK. 1953 - Fungi of Assam. Journal University of Gauhati 4, 287-312.

Bhavani Devi S 1995 - Mushroom flora of Kerala. In: Advances in Hortriculture, Vol. 13- Mushrooms (eds KL Chadha, SR Sharma). Malhotra Publishing House, New Delhi, pp 277-316.

Bose SR 1920 - Records of Agaricaceae from Bengal. The journal of the Asiatic Society of Bengal 16, 347-354.

Bose SR, Bose AB. 1940 - An account of edible mushrooms of India. Science \& Culture 6, 141-149.

Butler EJ, Bisby GR. 1931 - The Fungi of India. Imperial (Indian) Council of Agricultural Research, Calcutta, Science Monograph 1, XVIII, pp 237.

Dhancholia S, Sinha MP. 1990 - Additional studies on Agarics of Orissa II. Geobios New Reports 9, 108-113.

Dutta AK, Pradhan P, Roy A, Acharya K. 2011 - Volvariella of West Bengal, India I. Researcher 3(5), 13-17.

Garcha HS, Kalra KL. 1977 - Weed Mushrooms. Symposium on Recent Researches in Plant Sciences, 20-22 January, Department of Botany, Punjabi University, Patiala. pp. 8 (abstr).

Ghosh RN, Pathak NC, Singh BP. 1974 - Studies on Indian Agaricales II. The Proceedings of the National Academy of Sciences, India 44(B), 125-128.

Ghosh RN, Pathak NC, Singh MS. 1976 - The genus Chlorophyllum in India. Indian Phytopathology 29(1), 50-53.

Ghosh RN, Pathak NC, Tiwari T. 1967 - Studies on Indian Agaricales. Indian Phytopathology 20, 237-242.

Ginai MA 1936 - Further contribution to knowledge of Indian coprophilous fungi. Journal of the Indian Botanical Society 15, 269-284.

Gogoi R, Manjumder D, Puzari KC. 2000 - New additions to Mushroom flora of Assam. Mushroom Research 9(1), 55.

Hennings P 1900 - Fungi Indiae Orientalis. Hedwigia 39, 150-153.

Hennings P. 1901 - Fungi Indiae Orientalis II. Hedwigia 40, 323-342.

Hopple JS, Vilgalys R. 1999 - Phylogenetic relationships in the mushroom genus Coprinus and darkspored allies based on sequence data from the nuclear gene coding for the large ribosomal subunit RNA: divergent domains, outgroups, and monophyly. Molecular Phylogenetics and Evolution 13, 1-19.

Justo A, Minnis AM, Ghignone S, Menolli Jr N, Capelari M, Rodríguez O, Malysheva E, Contu M, Vizzini A. 2010a - Species recognition in Pluteus and Volvopluteus (Pluteaceae, Agaricales): morphology, geography and phylogeny. Mycological Progress 10, 453-479.

Justo A, Vizzini A, Minnis AM, Menolli Jr N, Capelari M, Rodríguez O, Malysheva E, Contu M, Ghignone S, Hibbett DS. 2010b - Phylogeny of the Pluteaceae (Agaricales, Basidiomycota): Taxonomy and character evolution. Fungal Biology 30, 1-20. 
Kannaiyan S, Ramasamy K. 1980 - A Hand Book of Edible Mushrooms. Today and Tomorrow's Printers and Publishers, New Delhi, pp 104.

Karun NC, Sridhar KR. 2015 - Elephant dung-inhabiting macrofungi in the Western Ghats. Current Research in Environmental \& Applied Mycology 5(1), 60-69, Doi 10.5943/cream/5/1/8.

Kaul TN 1978 - Mushroom cultivation and rural development. Indian Mushroom Science 1, 1-13.

Kaul TN, Kachroo JL. 1974 - Common edible mushrooms of Jammu and Kashmir. Journal of the Bombay Natural History Society 71, 26-31.

Kaur A, Atri NS, Kaur M. 2013a - A new variety of Rhodocybe popinalis (Entolomataceae, Agaricales) from coprophilous habitats of India. Journal on New Biological Reports 2(3), 260 263.

Kaur A, Atri NS, Kaur M. 2013b - A new species of Psathyrella (Psathyrellaceae, Agaricales) collected on dung from Punjab, India. Journal on New Biological Reports 2(3), 275-280.

Kaur A, Atri NS, Kaur M. 2014a - Two new species of Panaeolus (Psathyrellaceae, Agaricales) from coprophilous habitats of Punjab, India. Journal on New Biological Reports 3(2), 125-132.

Kaur A, Atri NS, Kaur M. 2014b - Two new species of Agaricus (Agaricaceae, Agaricales) collected on dung from Punjab, India. Kavaka 42, 20-24.

Kaur A, Atri NS, Kaur M. 2014c - Diversity of coprophilous species of Panaeolus (Psathyrellaceae, Agaricales) from Punjab, India. Biodiversitas 15(2), 115-130, Doi 10.13057/biodiv/d150202.

Kaur A, Atri NS, Kaur M. 2014d - Taxonomic study on species of Agrocybe (Strophariaceae, Agaricales) collected on dung from Punjab, India. Kavaka 43, 46-49.

Kaur A, Kaur M, Atri NS. 2013c - Protostropharia semiglobata var. punjabensis: A new coprophilous agaric from India. Kavaka 41, 11-14.

Kaushal SC, Grewal K. 1992 - Coprophilous fungi from Chattbir. National Symposium on Botanical Research Trends and Achievements, 30-31 March, 1992, Department of Botany, Punjab University, Chandigarh, pp 19-20 (abst).

Kew Mycology 2013 - Checklist and database of the British Basidiomycota. Royal Botanic Gardens, Kew.

Krishnamurthy V, Verma JP. 1974 - Preliminary studies on Boll rot of cotton in India. Cotton Growing Review 51, 26-227.

Kumar TKA, Manimohan P. 2009 - The genus Lepiota (Agaricales, Basidiomycota) in Kerala state, India. Mycotaxon 107, 105-138.

Lakhanpal TN 1986 - Hallucinogenic mushrooms In: Souvenir of Mushrooms, National Centre for Mushroom Research and Training, Solan.

Lakhanpal TN 1993 - The Himalayan Agaricales - Status of systematics. Mushroom Research 2(1), 1 $-10$.

Mahju NA 1933 - A Contribution to our knowledge of Indian coprophilous fungi. Journal of the Indian Botanical Society 12, 153-164.

Manimohan PK, Thomas A, Nisha VS. 2007 - Agarics on elephant dung in Kerala State, India. Mycotaxon 99, 147-157.

Manjula B 1980 - Taxonomic studies on South Indian Agaricales. PhD. Thesis, University of Madras, Madras.

Manjula B 1983 - A revised list of Agaricoid and Boletoid Basidiomycetes from India and Nepal. The Proceedings of the National Academy of Sciences, India, Plant Science 92, $81-213$.

Massee G 1901 - Fungi Exotici III. Bulletin of Miscellaneous Information of the Royal Botanical Gardens, Kew, 150-169.

Mohanan C 2011 - Macrofungi of Kerala. KFRI Handbook \# 27, Kerala Forest Research Institute, Peechi, Kerala, India.

Moses ST 1948 - A preliminary report on the mushrooms of Baroda. Department of Fisheries, Baroda, Bulletin 14, 1-3.

Natarajan K 1977 - A new species of Termitomyces from India. Current Science 46(19), 679-680.

Natarajan K 1978 - South Indian Agaricales VI. Kavaka 6, 65-70.

Natarajan K, Manjula B. 1981 - South Indian Agaricales XIV. Indian Journal of Botany 4, 50-59. 
Natarajan K, Raaman N. 1983 - South Indian Agaricales. Bibliotheca Mycologica 89, 1-203.

Natarajan K, Raaman N. 1984 - South Indian Agaricales- A preliminary study on some dark spored species. International Books and Periodicals Supply Services, New Delhi, pp 204.

Noordeloos ME 2009 - The genus Deconica (W. G. Sm.) P. Karst. in Europe - new combinations. Österreichische Zeitschrift für Pilzkunde 18, 207-210.

Noordeloos ME, Vrinda KB, Manimohan P. 2007 - On two remarkable brown-spored agarics from Kerala state, India. Fungal Diversity 27, 145-155.

Pal A, Mukherjee SK. 1977 - Cultivation of white mushroom Agaricus bisporus. Bulletin of the Botanical Society of Bengal 31, 43-45.

Patel MK, Kamat MN. 1935 - The Fungi of Bombay 8, 1-56.

Pathak NC, Ghosh RN, Singh MS. 1978 - The genus Volvariella in India. Indian Mushroom Science 1, 295-303.

Patil BD, Jadhav SW, Sathe AV. 1995 - Mushroom flora of Maharashtra. In: Advances in Hortriculture Vol. 13- Mushrooms (eds KL Chadha, SR Sharma). Malhotra Publishing House, New Delhi, pp 317-328.

Pegler DN, Vanhaecke M. 1994 - Termitomyces of Southeast Asia. Kew Bulletin 49(4), 717-735.

Pradeep CK, Vrinda KB, Mathew S, Abraham TK. 1998 - The genus Volvariella in Kerala state, India. Mushroom Research 7(2), 53-62.

Pushpa H, Purushothama KB. 2011 - Leucocoprinus Pat. (Agaricaceae, Agaricales, Basidiomycota) in Bengaluru, Karnataka state, India. World Applied Sciences Journal 14(3), 470-475. ISSN $1818-4952$.

Pushpa H, Purushothama KB. 2012 - Biodiversity of mushrooms in and around Bangalore (Karnataka), India. American-Eurasian Journal of Agricultural \& Environmental Sciences 12(6), 750-759.

Rawla GS, Sarwal BM, Arya S. 1982 - Agarics new to India I. Nova Hedwigia 36, 433-43.

Rea C 1922 - British Basidiomycetaceae: A Handbook to the Larger British Fungi. Cambridge University Press, Cambridge, England, pp 799.

Redhead SA, Vilgalys R, Moncalvo JM, Johnson J, Hopple JS. 2001 - Coprinus Pers. and the disposition of Coprinus species sensu lato. Taxon 50, 203-241.

Saini MK, Singh Y, Kaur H, Atri NS. 2008-2009 - The genus Volvariella Speg. from North India. Journal of Punjab Academy of Sciences 5-6(1\&2), 52-56.

Saini SS, Atri NS, Gupta AK. 1991 - Additional studies on North-West Indian Agarics. In: Indian Mushrooms, (ed MC Nair). Kerala Agricultural University, Velenikkara, India, pp 7-12.

Saini SS, Atri NS, Singh K. 1983 - Volvariella hypopithys- a new record for India. Indian Phytopathology 36, 180-182.

Saini SS, Atri NS. 1993 - North Indian Agaricales-IV. Indian Journal of Mycology and Plant Pathology 23(3), 250-54.

Saini SS, Atri NS. 1995 - Mushroom flora of Punjab. In: Advances in Horticulture Vol 13Mushrooms (eds KL Chadha, SR Sharma). Malhotra Publishing House, New Delhi, India, pp 375-386.

Sarwal BM, Rawla GS. 1983 - Taxonomic studies on Indian Agarics-III. Bibliotheca Mycologica 91, 541-548.

Sathe AV, Deshpande S. 1980 - Agaricales (Mushrooms) of Maharashtra, MACS Monograph 1, 9-42.

Sathe AV, Rahalkar SR. 1978 - Agaricales from South-West India-III. In: Indian Mushroom Science, (eds CK Atal, BK Bhat, TN Kaul). Indo-American Literature House, USA.

Sehgal BK 1978 - Mushroom cultivation at Kasauli, Himachal Pradesh. Indian Mushroom Science 1, 29-34.

Sharma AD, Jandaik CL, Munjal RL, Seth PK. 1978 - Some fleshy fungi from Himachal Pradesh-I. Indian Journal of Mushrooms 4, 1-4.

Singh J, Mehrotra BS 1974 - A survey of the gilled mushrooms in India, Beihefte zur Nova Hedwigia 47, 511-529.

Srivastava BS 1978 - Marketing of white mushrooms. Indian Mushroom Science 1, 113-117.

Thapa CD, Kumar S, Jandaik CL, Seth PK. 1977 - Some weed fungi occurring in mushroom (Agaricus 
bisporus) bed-I. Indian Journal of Mushrooms 3, 27-28.

Thomas KA, Hausknecht A, Manimohan P. 2001 - Bolbitiaceae of Kerala State, India: New species and new and noteworthy records. Österreichische Zeitschrift für Pilzkunde 10, 87-114.

Thomas KA, Manimohan P. 2002 - The genus Psilocybe in Kerala state, India. Mycotaxon 83, 195207.

Thomas KA, Manimohan P. 2003 - The genus Agrocybe in Kerala state, India. Mycotaxon 86, 317333.

Trivedi TK 1972 - Agaricales of Nagpur-I. The Botanique 3, 53-59.

Vasudeva RS 1960 - The Fungi of India (Revised), ICAR, New Delhi, pp. 255.

Vellinga EC 2002 - New combinations in Chlorophyllum. Mycotaxon 83, 415-417.

Verma RN, Singh GB, Singh MS. 1995 - Mushroom Flora of North Eastern Hills. In: Advances in Horticulture Vol 13- Mushrooms (eds KL Chadha, SR Sharma). Malhotra Publishing House, New Delhi, India, pp 329-349.

Vrinda KB, Pradeep CK, Mathew S, Abraham TK. 1999 - Agaricales from Western Ghats-VI. Indian Phytopathology 52(2), 198-200.

Watling R, Gregory NM. 1980 - Larger fungi from Kashmir. Nova Hedwigia 32, 493-564.

Wolport S 1999 - A New History of India (6 ${ }^{\text {th }}$ ed.), Oxford University Press. ISBN 978-0-19-512877-2 